\title{
LINEAR INTEGRO-DIFFERENTIAL EQUATIONS WITH A BOUNDARY
}

\section{CONDITION*}

BY

MINFU TAH HU

CONTENTS

1. Introduction and Notations $\ldots \ldots \ldots \ldots \ldots \ldots \ldots \ldots \ldots \ldots \ldots \ldots \ldots \ldots \ldots \ldots$

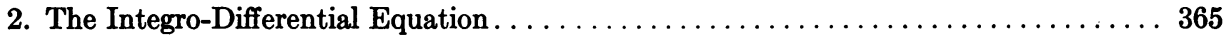

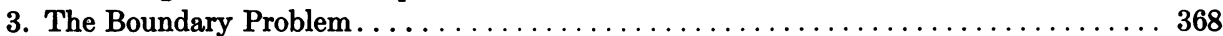

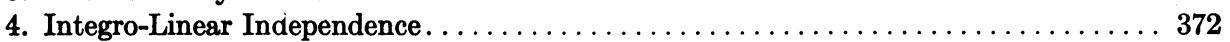

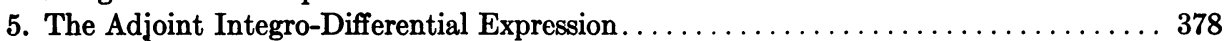

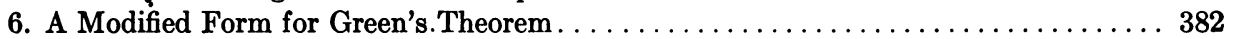

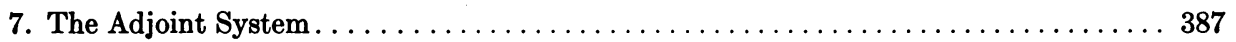

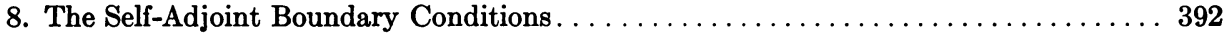

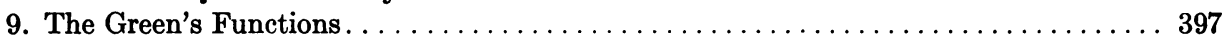

\section{Introduction and Notations}

It is a well-known fact that linear integral equations of the first and second kinds may be regarded as the limiting cases, as $n$ becomes infinite, of systems of $n$ linear algebraic equations in $n$ variables.

The same idea of passing to a limit suggests that one treat the integrodifferential equation

$$
\text { (A) } \frac{\partial u}{\partial x}+\phi(x, s) u(x, s)+\int_{a}^{\beta} \psi\left(\begin{array}{c}
s \\
x t
\end{array}\right) u(x, t) d t=\lambda(x, s)
$$

as the limit of a system of $n$ linear differential equations of the first order of the form $†$

* Presented to the Society, December 28, 1917. The problem treated in this paper was first suggested to me by Professor W. A. Hurwitz, to whom, and to Professor M. Bôcher, I tender my grateful acknowledgment for constant help, suggestions, and criticisms.

$\dagger$ For the system $(a)$ when all the equations are homogeneous, a different integro-differential

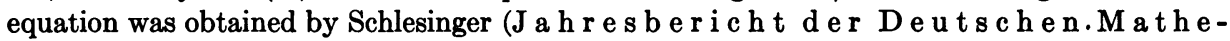
matiker-Vereinigung, vol. 24 (1915), p. 84) by means of a process involving certain changes of the form of the equations $(a)$ before passing to the limit. The equation thereby obtained differs from $(A)$ in that the variable $x$ is complex and all functions involved are analytic functions in $x$, that the functions $u$ and $\lambda$ contain another variable $r$ of the same class as $s$, and that

$$
\phi(x, s) \equiv 0 \quad \text { and } \quad \lambda(x, s) \equiv \psi\left(\begin{array}{c}
s \\
x r
\end{array}\right) .
$$




$$
\frac{d u_{1}(x)}{d x}+l_{11}(x) u_{1}(x)+\cdots+l_{1 n}(x) u_{n}(x)=\lambda_{1}(x),
$$

$$
\frac{d u_{n}(x)}{d x}+l_{n 1}(x) u_{1}(x)+\cdots+l_{n n}(x) u_{n}(x)=\lambda_{n}(x) .
$$

We shall have occasion to adjoin to $(A)$ a boundary condition of the type

$$
\begin{aligned}
\alpha(s) u(a, s)+\beta(s) u(b, s)+\int_{a}^{\beta}[ & A(s, r) u(a, r) \\
& +B(s, r) u(b, r)] d r=\gamma(s) .
\end{aligned}
$$

This we shall call a two-point boundary condition since it involves the two values $a$ and $b$ of the variable $x$. This is obviously the limiting form of the system of linear boundary conditions usually attached to the finite differential system $(a)$, as we let the number of equations increase indefinitely.

Throughout this paper, all variables entering will be real. These variables may be conveniently divided into two classes corresponding respectively to the first and second arguments of the unknown function $u$ in the equations $(A),(B)$. The first class of variables is denoted by such letters as $x, y, z$, $\xi, \eta, \zeta$, and they take on the values in the closed interval

$$
I: \quad a \leqq x \leqq b .
$$

We shall speak of this in the future simply as the interval $I_{x}$, the subscript $x$ indicating the variable referred to.

The second class of variables is usually denoted by the letters $s, t, r, \sigma, \tau, \rho$, which take on the values in the interval

$$
J: \quad \alpha \leqq s \leqq \beta .
$$

In the case of functions of two or more variables, each of which is confined to one of the intervals $I$ and $J$, we interpret the different variables as rectangular coördinates. For instance, the unknown function $u(x, s)$ will be supposed to be defined in the rectangle

$$
I_{x} J_{s}: \quad a \leqq x \leqq b, \quad \alpha \leqq s \leqq \beta .
$$

In case the variables belong to the same class, we shall have square regions $I_{x y}$ or $J_{s t}$. Likewise, for functions involving more than two variables we have

Then Schlesinger considered also the associated homogeneous equation of the type $(A)$ whose solutions are made dependent on the solutions of the former equation. These equations were also treated in a similar fashion in the notation of general analysis by $T$. H. Hildebrandt (these Transactions, vol. 18 (1917), p. 73). [After the manuscript of the present paper was in the hands of the editors of the Transactions, I was informed by them that a second paper by Hildebrandt was to appear shortly in the Transactions. See vol. 19 (1918), p. 97.] 
such regions as $I_{x y} J_{s}, I_{x y} J_{s t}$, etc. All these intervals and regions will be understood to be closed.

To simplify our work, we shall assume, unless otherwise stated, that all functions considered are real and continuous (and therefore bounded) in the respective regions in which they are defined. By a solution of the equations $(A),(B)$, or any other equation under consideration, we understand, without further specification, a continuous function. A solution of the equations $(A),(B)$, possesses a continuous first derivative with respect to its first argument. A solution which is identically zero will be termed a trivial solution.

\section{The Integro-Differential Equation}

The integro-differential equation

(A) $\frac{\partial u(x, s)}{\partial x}+\phi(x, s) u(x, s)+\int_{a}^{\beta} \psi\left(\begin{array}{c}s \\ x t\end{array}\right) u(x, t) d t=\lambda(x, s)$

may be reduced, by means of the transformation*

$$
u(x, s)=e^{-\int_{y}^{x} \varphi(\xi, s) d \xi} v(x, s),
$$

where $y$ is regarded as a fixed point in $I_{x}$, to the equation

$$
\begin{aligned}
\frac{\partial v(x, s)}{\partial x}+\int_{a}^{\beta} \psi\left(\begin{array}{c}
s \\
x t
\end{array}\right) e^{-\int_{y}^{x}[\varphi(\xi, t)-\varphi(\xi, s)] d \xi} & \\
& \times v(x, t) d t=e^{\int_{y}^{x} \varphi(\xi, s) d \xi} \lambda(x, s) .
\end{aligned}
$$

This equation is the special case of $(A)$ in which the second term of the first member is lacking. Let us write for convenience

so that

$$
R\left(\begin{array}{l}
x s \\
y
\end{array}\right)=e^{-\int_{y}^{x} \varphi(\xi, s) d \xi},
$$

$$
R\left(\begin{array}{l}
x s \\
y
\end{array}\right) R\left(\begin{array}{l}
y s \\
\xi
\end{array}\right)=R\left(\begin{array}{l}
x s \\
\xi
\end{array}\right) .
$$

Changing $x$ in (1) into $\xi$ and integrating from $y$ to $x$, we find

$$
\begin{aligned}
v(x, s)=v(y, s)+\int_{y}^{x} R\left(\begin{array}{l}
y s \\
\xi
\end{array}\right) \lambda(\xi, s) d \xi \\
\quad-\int_{y}^{x} \int_{a}^{\beta} \psi\left(\begin{array}{c}
s \\
\xi t
\end{array}\right) R\left(\begin{array}{l}
y s \\
\xi
\end{array}\right) R\left(\begin{array}{l}
\xi t \\
y
\end{array}\right) v(\xi, t) d t d \xi .
\end{aligned}
$$

* This was pointed out to me by Professor Birkhoff. 
We may now transform back to $u(x, s)$, getting

$$
\begin{aligned}
u(x, s)=R\left(\begin{array}{l}
x s \\
y
\end{array}\right) u(y, s)+\int_{y}^{x} R\left(\begin{array}{c}
x s \\
\xi
\end{array}\right) \lambda(\xi, s) d \xi \\
+\int_{y}^{x} \int_{a}^{\beta}\left[-R\left(\begin{array}{c}
x s \\
\xi
\end{array}\right) \psi\left(\begin{array}{c}
s \\
\xi t
\end{array}\right)\right] u(\xi, t) d t d \xi .
\end{aligned}
$$

This is a special case of the equation

$$
u(x, s)=f(x, s)+\int_{y}^{x} \int_{\alpha}^{\beta} \theta\left(\begin{array}{l}
x s \\
\xi t
\end{array}\right) u(\xi, t) d t d \xi .
$$

Let us then consider (4).

The function $\theta\left(\begin{array}{c}x: \\ \xi i\end{array}\right)$ (called the kernel of the equation) will be supposed to be continuous in $I_{x \xi} J_{s t}$. In its appearance, the equation is intermediate between the Volterra and the Fredholm types; but it behaves like an equation of the Volterra type because of the variable limit of the first integral. Since Volterra's method may be applied almost word for word,* we shall give here only the results.

We are led by the method of successive substitutions to the consideration of the series

$$
\theta_{1}\left(\begin{array}{c}
x s \\
\xi t
\end{array}\right)+\theta_{2}\left(\begin{array}{c}
x s \\
\xi t
\end{array}\right)+\theta_{3}\left(\begin{array}{c}
x s \\
\xi t
\end{array}\right)+\cdots
$$

where

$$
\begin{aligned}
\theta_{1}\left(\begin{array}{c}
x s \\
\xi
\end{array}\right) & =\theta\left(\begin{array}{c}
x s \\
\xi t
\end{array}\right), \\
\theta_{n}\left(\begin{array}{c}
x s \\
\xi
\end{array}\right) & =\int_{\xi}^{x} \int_{a}^{\beta} \theta_{n-1}\left(\begin{array}{l}
x s \\
\eta r
\end{array}\right) \theta_{1}\left(\begin{array}{c}
\eta r \\
\xi t
\end{array}\right) d r d \eta .
\end{aligned}
$$

The series (5) converges absolutely and uniformly in $I_{x \xi} J_{s t}$, thus representing a bounded continuous function, $\theta\left(\begin{array}{c}x \\ \xi\end{array}\right)$, which shall be called the resolvent function of the kernel $\theta\left(\begin{array}{l}x s \\ \xi\end{array}\right)$.

The kernel and the resolvent function satisfy the resolvent formulæ

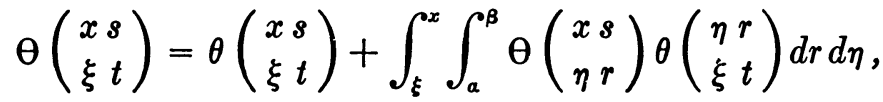

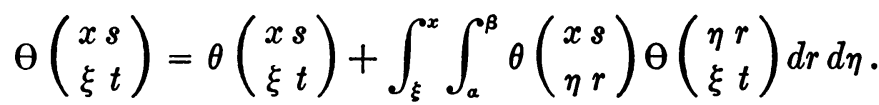

We now readily establish the

* See Volterra: Leçons sur les équations intégrales, p. 74, where an equation is treated which is identical with (4), except that $\beta$ is replaced by the variable $s$. The possibility of using the same method when one of the upper limits is constant was pointed out to me by Professor W. A. Hurwitz. 
LEMma. If $\theta\left(\begin{array}{c}x \\ \xi\end{array}\right)$ is continuous in $I_{x \xi} J_{s t}$ and $f(x, s)$ is continuous in $I_{x} J_{s}$, then the equation (4) has one and only one solution, namely

$$
u(x, s)=f(x, s)+\int_{y}^{x} \int_{a}^{\beta} \theta\left(\begin{array}{c}
x s \\
\xi \\
t
\end{array}\right) f(\xi, t) d t d \xi .
$$

Returning now to the equation $\left(A^{\prime}\right)$, let

$$
\begin{aligned}
\theta\left(\begin{array}{l}
x s \\
\xi t
\end{array}\right) & =-R\left(\begin{array}{l}
x s \\
\xi
\end{array}\right) \psi\left(\begin{array}{c}
s \\
\xi t
\end{array}\right), \\
f(x, s) & =R\left(\begin{array}{l}
x s \\
y
\end{array}\right) u(y, s)+\int_{y}^{x} R\left(\begin{array}{l}
x s \\
\xi
\end{array}\right) \lambda(\xi, s) d \xi,
\end{aligned}
$$

where $y$ is regarded as a fixed point in $I_{x}$. Thus we obtain by the lemma, for each assigned function $u(y, s)$, a unique solution of $\left(A^{\prime}\right)$ or $(A)$, which, if we let

$$
S\left(\begin{array}{l}
x s \\
y t
\end{array}\right)=\int_{y}^{x} \theta\left(\begin{array}{l}
x s \\
\xi
\end{array}\right) R\left(\begin{array}{l}
\xi t \\
y
\end{array}\right) d \xi
$$

may be put into the form

$$
\begin{aligned}
u(x, s)=R & \left(\begin{array}{l}
x s \\
y
\end{array}\right) u(y, s)+\int_{a}^{\beta} S\left(\begin{array}{l}
x s \\
y t
\end{array}\right) u(y, t) d t \\
& +\int_{y}^{x}\left[R\left(\begin{array}{c}
x s \\
\xi
\end{array}\right) \lambda(\xi, s)+\int_{a}^{\beta} S\left(\begin{array}{c}
x s \\
\xi t
\end{array}\right) \lambda(\xi, t) d t\right] d \xi .
\end{aligned}
$$

Hence, we have

TheоRem I. The integro-differential equation $(A)$ possesses one and only one solution which reduces to the assigned initial function $u(y, s)$ at the fixed point $y$ in $I_{x}$; this solution is given by the formulo (11).

Corollary I. If the integro-differential equation $(A)$ is homogeneous, $i$. e., if $\lambda(x, s) \equiv 0$, the solution has the form

$$
u(x, s)=R\left(\begin{array}{l}
x s \\
y
\end{array}\right) u(y, s)+\int_{a}^{\beta} S\left(\begin{array}{l}
x s \\
y t
\end{array}\right) u(y, t) d t .
$$

Corollary II. The function

$$
w\left(\begin{array}{l}
x s \\
y
\end{array}\right)=\int_{y}^{x}\left[R\left(\begin{array}{l}
x s \\
\xi
\end{array}\right) \lambda(\xi, s)+\int_{a}^{\beta} S\left(\begin{array}{l}
x s \\
\xi t
\end{array}\right) \lambda(\xi, t) d t\right] d \xi
$$

is a particular solution of the non-homogeneous equation $(A)$, corresponding to the initial function $u(y s) \equiv 0$.

Observe that the integrand of the expression $w\left(\begin{array}{l}x s \\ y\end{array}\right)$, when regarded as a function of $x$ and $s$, is a solution of the homogeneous equation $(A)$ for each constant value of $\xi$. Thus the particular solution $w\left(\begin{array}{l}x^{8} \\ y\end{array}\right)$ of the non-homo- 
geneous equation $(A)$ is built up from the solutions of the homogeneous equation by an integration. It is clear that every other solution of the nonhomogeneous equation is obtainable by adding to the particular solution $w$ a solution of the homogeneous equation.

We shall also have occasion to apply the following:

Corollary III. The function $\theta\left(\begin{array}{l}x \\ y \\ y\end{array}\right)$, when regarded as a function in $x$ and $s$, is a solution of the homogeneous equation $(A)$, corresponding to the initial function $\theta\left(\begin{array}{l}x \\ y\end{array} i\right)=-\psi\left(\begin{array}{l}y \\ y\end{array}\right)$ at $y$.

On account of the resolvent formula (6), we have

$$
\theta\left(\begin{array}{l}
y s \\
y t
\end{array}\right)=\theta\left(\begin{array}{l}
y s \\
y t
\end{array}\right)
$$

and, on account of the first formula (9) and formula (2),

$$
\theta\left(\begin{array}{c}
y s \\
y t
\end{array}\right)=-\psi\left(\begin{array}{c}
s \\
y t
\end{array}\right)
$$

Consequently, by combining (9), (14), (15),

$$
\theta\left(\begin{array}{l}
x s \\
y t
\end{array}\right)=R\left(\begin{array}{l}
x s \\
y
\end{array}\right) \theta\left(\begin{array}{l}
y s \\
y t
\end{array}\right)
$$

and because of (10) the equation (6) becomes

$$
\Theta\left(\begin{array}{l}
x s \\
y t
\end{array}\right)=R\left(\begin{array}{l}
x s \\
y
\end{array}\right) \Theta\left(\begin{array}{l}
y s \\
y t
\end{array}\right)+\int_{a}^{\beta} S\left(\begin{array}{l}
x s \\
y \sigma
\end{array}\right) \Theta\left(\begin{array}{l}
y \sigma \\
y t
\end{array}\right) d \sigma,
$$

which is a solution by Corollary I.

\section{The Boundary Problem}

Let us now take a linear integral boundary expression of the following type:

$$
U[u] \equiv \alpha(s) u(a, s)+\beta(s) u(b, s)
$$

$$
+\int_{a}^{\beta}[A(s, r) u(a, r)+B(s, r) u(b, r)] d r,
$$

where $\alpha(s), \beta(s)$ are continuous functions in $J_{s}, A(s, r), B(s, r)$ are continuous functions in $J_{s t}$, and $a, b$ are the end points of the interval $I_{x}$. Let us write from now on

(2) $L[u] \equiv \frac{\partial u(x, s)}{\partial x}+\phi(x, s) u(x, s)+\int_{a}^{\beta} \psi\left(\begin{array}{c}s \\ x t\end{array}\right) u(x, t) d t$.

We shall consider the integro-differential boundary problems 
and

$$
\text { (A) } L[u]=\lambda(x, s), \quad(B) \quad U[u]=\gamma(s)
$$

$$
\left(A_{0}\right) \quad L[u]=0, \quad\left(B_{0}\right) \quad U[u]=0 .
$$

It has been seen that all solutions of the non-homogeneous and the homogeneous equations $(A),\left(A_{0}\right)$ are of the forms

$$
\begin{gathered}
u(x, s)=w\left(\begin{array}{l}
x s \\
y
\end{array}\right)+R\left(\begin{array}{l}
x s \\
y
\end{array}\right) u(y, s)+\int_{a}^{\beta} S\left(\begin{array}{l}
x s \\
y t
\end{array}\right) u(y, t) d t \\
u(x, s)=R\left(\begin{array}{l}
x s \\
y
\end{array}\right) u(y, s)+\int_{\alpha}^{\beta} S\left(\begin{array}{l}
x s \\
y t
\end{array}\right) u(y, t) d t
\end{gathered}
$$

respectively, where $y$ is a fixed point in the interval $I_{x}$ at which the initial function $u(y, s)$ is to be assigned. Both $y$ and the continuous function $u(y, s)$ are arbitrary. But in order to satisfy the boundary condition, it is clear that the initial function must be suitably chosen.

Substituting in (1) the value of $u(x, s)$ from (3), we find that the boundary condition $(B)$ reduces to

(5) $g(y, s) u(y, s)+\int_{a}^{\beta} G\left(\begin{array}{c}s \\ y t\end{array}\right) u(y, t) d t=\gamma(s)-U\left[w\left(\begin{array}{l}x s \\ y\end{array}\right)\right]$, where

$$
\begin{aligned}
g(y, s) & =\alpha(s) R\left(\begin{array}{l}
a s \\
y
\end{array}\right)+\beta(s) R\left(\begin{array}{l}
b s \\
y
\end{array}\right) \\
G\left(\begin{array}{r}
s \\
y t
\end{array}\right) & =A(s, t) R\left(\begin{array}{l}
a t \\
y
\end{array}\right)+B(s, t) R\left(\begin{array}{l}
b t \\
y
\end{array}\right)+U\left[S\left(\begin{array}{l}
x s \\
y
\end{array}\right)\right] .
\end{aligned}
$$

This is an integral equation for determining the initial function $u(y, s)$.

Likewise, the equation $\left(B_{0}\right)$ of the homogeneous system reduces to the homogeneous integral equation

$$
g(y, s) u(y, s)+\int_{a}^{\beta} G\left(\begin{array}{c}
s \\
y t
\end{array}\right) u(y, t) d t=0 .
$$

Now we impose the further condition that $\alpha(s)$ and $\beta(s)$ be such that $g(y, s)$ do not vanish at any point of $J_{s}$, so that the equations (5) and (7) may be reduced to integral equations of the second kind. Let us, then, examine this condition a little further by allowing the point $y$ in the expression $g(y, s)$ to vary in $I_{y}$. Now

$$
g(y, s)=\alpha(s) e^{-\int_{y}^{a} \varphi(\xi, s) d \xi}+\beta(s) e^{-\int_{y}^{b} \varphi(\xi, s) d \xi}
$$

If $g(y, s) \neq 0$ for a particular value of $y$, then

$$
\alpha(s)+\beta(s) e^{-\int_{a}^{b} \varphi(\xi, s) d \xi} \neq 0,
$$


i. e.,

$$
\alpha(s)+\beta(s) R\left(\begin{array}{l}
b s \\
a
\end{array}\right) \neq 0 .
$$

Conversely, if $(C)$ is fulfilled, then we shall have $g(y, s) \neq 0$ throughout $J$. for each value of $y$ in $I_{y}$. Thus the condition $(C)$ and the condition $g(y, s)$ $\neq 0$ are equivalent conditions, but it should be noticed that condition $(C)$ does not involve $y$. Hereafter we shall always assume that $(C)$ is fulfilled.

Under the condition $(C)$ the equations (5) and (7) become

$$
\begin{gathered}
u(y, s)=F(y, s)+\int_{\alpha}^{\beta} K\left(\begin{array}{c}
s \\
y t
\end{array}\right) u(y, t) d t, \\
u(y, s) \cdot=\int_{\alpha}^{\beta} K\left(\begin{array}{r}
s \\
y t
\end{array}\right) u(y, t) d t,
\end{gathered}
$$

where

$$
\begin{aligned}
K\left(\begin{array}{c}
s \\
y t
\end{array}\right) & =-\frac{G\left(\begin{array}{c}
s \\
y t
\end{array}\right)}{g(y, s)}, \\
F(y, s) & =\frac{\gamma(s)-U\left[w\left(\begin{array}{l}
x s \\
y
\end{array}\right)\right]}{g(y, s)} .
\end{aligned}
$$

The problem of solving the system $(A, B)$ or $\left(A_{0}, B_{0}\right)$ then reduces to the determination of the initial function $u(y, s)$ from the equation $\left(5^{\prime}\right)$ or $\left(7^{\prime}\right)$. The initial function so determined will give the solution of the system upon substituting into the equation (3) or (4).

As in the theory of differential equations, the homogeneous system $\left(A_{0}, B_{0}\right)$ is said to be incompatible if it possesses no non-trivial solution; it is said to have compatibility of the kth order or index $k$ if there are precisely $k$ linearly independent solutions.

Suppose $u_{1}(x, s), \cdots, u_{n}(x, s)$ are linearly dependent solutions of the homogeneous system $\left(A_{0}, B_{0}\right)$. Then there exist constants $c_{1}, \cdots, c_{n}$, not all zero, such that

$$
c_{1} u_{1}(x, s)+\cdots+c_{n} u_{n}(x, s)=0
$$

identically in $I_{x} J_{s}$; in particular,

$$
c_{1} u_{1}(y, s)+\cdots+c_{n} u_{n}(y, s)=0
$$

for a particular value $y$ in $I_{x}$. Conversely, if the initial functions $u_{i}(y, s)$ are linearly dependent, the solutions of the system formed by means of (4) will be linearly dependent. Hence

Theorem I. Under the condition $(C)$, a necessary and sufficient condition 
that the homogeneous system $\left(A_{0}, B_{0}\right)$ have index $k$ is that the integral equation ( $\left.7^{\prime}\right)$ have index* $k$.

Theorem I'. A necessary and sufficient condition that the system $\left(A_{0}, B_{0}\right)$, subject to the condition $(C)$, be incompatible is that the Fredholm determinant $D(y)$ of the kernel $K\left(\begin{array}{c}s \\ y_{t}\end{array}\right)$ of the equation $\left(7^{\prime}\right)$ does not vanish $\dagger$; if $D(y)$ vanishes, the index of the system is finite.

Since the condition $(C)$ does not depend on $y$, the kernel $K\left(\begin{array}{l}y_{i} \\ i\end{array}\right)$ of $\left(5^{\prime}\right)$ and $\left(7^{\prime}\right)$ and the Fredholm determinant $D(y)$ exist for every $y$ in $I_{y}$. Consequently, if $D\left(y_{0}\right) \neq 0$, the homogeneous system $\left(A_{0}, B_{0}\right)$ will be incompatible and therefore the equation $\left(7^{\prime}\right)$ can have only the trivial solution $u(y, s) \equiv 0$ for every $y$. Hence

Theorem II. The Fredholm determinant, $D(y)$, of the equations $\left(5^{\prime}\right)$ and $\left(7^{\prime}\right)$ either vanishes everywhere in $I_{y}$ or else vanishes nowhere.

Every solution of $\left(A_{0}, B_{0}\right)$ becomes, when $x$ is changed into $y$, a solution of $\left(7^{\prime}\right)$. Conversely, however, a solution of $\left(7^{\prime}\right)$ will not, in general, when $y$ is changed to $x$, become a solution of $\left(A_{0}, B_{0}\right)$, as is seen by the fact that a solution of $\left(7^{\prime}\right)$ may be multiplied by an arbitrary function of $y$ while a solution of $\left(A_{0}, B_{0}\right)$ cannot be multiplied by an arbitrary function of $x$. Consequently, $\left(A_{0}, B_{0}\right)$ is not, in general, equivalent to $\left(7^{\prime}\right)$. It is, however, equivalent in the special case in which $\left(A_{0}, B_{0}\right)$ is incompatible, since then $\left(7^{\prime}\right)$ also has no solution except zero. In this case $(A, B)$ has one and only one solution, which must, therefore, be the unique solution of $\left(5^{\prime}\right)$. Hence

Theorem III. The systems $(A, B),\left(A_{0}, B_{0}\right)$ are respectively equivalent to the integral equations $\left(5^{\prime}\right),\left(7^{\prime}\right)$ whenever the homogeneous system $\left(A_{0}, B_{0}\right)$ is incompatible; when $\left(A_{0}, B_{0}\right)$ is compatible, they are equivalent to $\left(5^{\prime}\right),\left(7^{\prime}\right)$ together with the auxiliary equations (3), (4) respectively.

Corollary. When the homogeneous system $\left(A_{0}, B_{0}\right)$ is incompatible, the non-homogeneous system $(A, B)$ has a unique solution, which is given by

$$
u(x, s)=F(x, s)+\int_{a}^{\beta} Q\left(\begin{array}{c}
s \\
x t
\end{array}\right) F(x, t) d t,
$$

where $Q\left({ }_{x} i\right)$ is the resolvent function of the kernel $K\left(x_{x} i\right)$ of the equations $\left(5^{\prime}\right),\left(7^{\prime}\right)$. When the homogeneous system is compatible, the non-homogeneous system $(A, B)$ possesses solutions if and only if

$$
\int_{a}^{\beta} \phi_{i}(x, s) F(x, s) d s=0
$$

for all solutions $\phi_{i}(x, s)$ of the equation

$$
\phi(x, s)=\int_{a}^{\beta} \phi(x, t) K\left(\begin{array}{r}
t \\
x s
\end{array}\right) d t .
$$

* Bôcher: An Introduction to the Study of Integral Equations, p. 45.

$\dagger$ Here the quantity $y$ is regarded as a constant, but arbitrary. 


\section{INTEGRo-LinEAR INDEPENDENCE}

A linear integral expression

$$
\begin{aligned}
U[u ; s] \equiv \alpha(s) u_{1}(s)+\beta(s) & u_{2}(s) \\
& \quad+\int_{a}^{\beta}\left[A(s, r) u_{1}(r)+B(s, r) u_{2}(r)\right] d r
\end{aligned}
$$

is said to be integro-linearly self-dependent in the interval $J_{s}$ (or simply selfdependent) if there exists a continuous function $c(s)$ in $J_{s}$, not identically zero, such that

$$
\int_{a}^{\beta} c(s) U[u ; s] d s=0
$$

for every pair of continuous functions $u_{1}(s), u_{2}(s)$; otherwise, it is said to be self-independent. Two linear integral expressions $U_{1}, U_{2}$ of the type (1) are said to be integro-linearly dependent (or simply dependent) if there exist continuous functions $c_{1}(s), c_{2}(s)$, not both identically zero, such that

$$
\int_{a}^{\beta}\left(c_{2}(s) U_{1}[u ; s]+c_{1}(s) U_{2}[u ; s]\right) d s=0
$$

for every pair of continuous functions $u_{1}(s)$ and $u_{2}(s)$; otherwise they are said to be independent.

The above definitions of independence and self-independence are obviously a generalization of the notion of linear independence for a system of algebraic expressions. We shall derive some necessary and sufficient conditions for such dependence.

ThEoREM I. A necessary and sufficient condition that the expression (1) be self-dependent is that the equations

$$
\begin{aligned}
& \alpha(s) c(s)+\int_{a}^{\beta} c(r) A(r, s) d r=0, \\
& \beta(s) c(s)+\int_{a}^{\beta} c(r) B(r, s) d r=0
\end{aligned}
$$

have a non-trivial solution $c(s)$ in common. This function $c(s)$ then satisfies (2), and conversely every function $c(s)$ which satisfies (2) also satisfies (4).

The theorem is an immediate result of (2) when we observe that $u_{1}(s)$ and $u_{2}(s)$ are arbitrary functions.

Corollary. A sufficient condition that $U[u ; s]$ be self-independent is that either one of the equations (4) possess no non-trivial solution.

Theorem II. When the $U$ of formula (1), $\S 3$, is self-dependent, the homogeneous system $\left(A_{0}, B_{0}\right)$ of $\S 3$, subject to the condition $(C)$ throughout $J_{s}$ as considered, has always a non-trivial solution. 
By hypothesis there exists a continuous function $c(s)$, not identically zero in $J_{s}$, which satisfies (2) and forms a common solution of the equations (4). Multiplying (4) by $R\left(\begin{array}{c}a b \\ y^{a}\end{array}\right)$ and $R\left(\begin{array}{l}b \\ y\end{array}\right)$ respectively and adding the results together, we have, by $(6), \S 3$,

$$
g(y, s) c(s)+\int_{a}^{\beta} c(r)\left\{G\left(\begin{array}{c}
r \\
y s
\end{array}\right)-U\left[S\left(\begin{array}{l}
x r \\
y s
\end{array}\right)\right]\right\} d r=0,
$$

and this, by (8), $\S 3$ and by (2) reduces to

$$
g(y, s) c(s)=\int_{a}^{\beta} c(r) g(y, r) K\left(\begin{array}{r}
r \\
y s
\end{array}\right) d r .
$$

As a solution of $\left(7^{\prime}\right), \S 3$, we have, then,

$$
u(y, s)=g(y, s) c(s) .
$$

Consequently the system $\left(A_{0}, B_{0}\right)$ has a non-trivial solution.

THEOREM III. If the homogeneous system $\left(A_{0}, B_{0}\right)$, subject to the condition $(C)$, is compatible and if the expression $U$ is self-independent, not every semihomogeneous system

$\left(A, B_{0}\right)$

$$
L[u]=\lambda(x, s), \quad U[u]=0
$$

possesses a solution.

Suppose the system $\left(A, B_{0}\right)$ does possess a solution for every $\lambda(x, s)$. Then, by the Corollary to Theorem III, $\S 3$,

$$
\int_{a}^{\beta} \phi_{i}(y, s) F(y, s) d s=0
$$

for every solution of the equation (11), $\S 3$. This equation reduces to

$$
\int_{a}^{\beta} \frac{\phi_{i}(y, s)}{g(y, s)} U\left[w\left(\begin{array}{l}
x s \\
y
\end{array}\right)\right] d s=0
$$

because of (8), $\S 3$. Expanding $U$ and substituting for $w\left({ }_{y}^{x}\right)$ its value from (13), § 2, we find

$$
\begin{aligned}
\int_{y}^{a} \int_{a}^{\beta}\left[R\left(\begin{array}{c}
a s \\
\xi
\end{array}\right) \Phi_{1}(y, s)+\int_{a}^{\beta} S\left(\begin{array}{c}
a s \\
\xi t
\end{array}\right) \Phi_{1}(y, t) d t\right] \lambda(\xi, s) d s d \xi \\
\quad+\int_{y}^{b} \int_{a}^{\beta}\left[R\left(\begin{array}{c}
b s \\
\xi
\end{array}\right) \Phi_{2}(y, s)+\int_{a}^{\beta} S\left(\begin{array}{c}
b s \\
\xi t
\end{array}\right) \Phi_{2}(y, t) d t\right] \lambda(\xi, s) d s d \xi=0,
\end{aligned}
$$

where

$$
\begin{aligned}
& \Phi_{1}(y, s)=\alpha(s) \frac{\phi_{i}(y, s)}{g(y, s)}+\int_{a}^{\beta} \frac{\phi_{i}(y, r)}{g(y, r)} A(r, s) d r, \\
& \Phi_{2}(y, s)=\beta(s) \frac{\phi_{i}(y, s)}{g(y, s)}+\int_{a}^{\beta} \frac{\phi_{i}(y, r)}{g(y, r)}-B(r, s) d r .
\end{aligned}
$$


We now find the following equations by assuming first that $\lambda(\xi, s)$ is zero when $\xi \geqq y$ while it is still arbitrary when $\xi<y$, and secondly that it is zero when $\xi \leqq y$ and arbitrary when $\xi>y$ :

$$
\begin{aligned}
& R\left(\begin{array}{c}
a s \\
\xi
\end{array}\right) \Phi_{1}(y, s)+\int_{a}^{\beta} S\left(\begin{array}{c}
a t \\
\xi s
\end{array}\right) \Phi_{1}(y, t) d t=0 \quad(a \leqq \xi \leqq y), \\
& R\left(\begin{array}{c}
b s \\
\xi
\end{array}\right) \Phi_{2}(y, s)+\int_{a}^{\beta} S\left(\begin{array}{c}
b t \\
\xi s
\end{array}\right) \Phi_{2}(y, t) d t=0 \quad(y \leqq \xi \leqq b) .
\end{aligned}
$$

Letting $\xi=a$ in the first equation and $\xi=b$ in the second, we obtain

$$
\Phi_{1}(y, s)=0, \quad \Phi_{2}(y, s)=0 .
$$

Consequently $\left[\phi_{i}(y, s)\right] /[g(y, s)]$ is a solution of (4) which does not vanish identically since $\phi_{i}$ may be assumed not to be identically zero. Therefore, by Theorem I, $U$ is self-dependent, which is contrary to hypothesis.

Theorem IV. A necessary condition that two linear expressions $U_{1}, U_{2}$ of the type (1) be independent is that each expression be self-independent.

Theorem V. A necessary and sufficient condition that the self-independent expressions $U_{1}, U_{2}$ be dependent on one another is that the equations

$$
\begin{aligned}
& \begin{array}{l}
\alpha_{1}(s) c_{2}(s)+\alpha_{2}(s) c_{1}(s)+ \\
+\int_{a}^{\beta}\left[c_{2}(r) A_{1}(r, s)\right. \\
\left.\quad+c_{1}(r) A_{2}(r, s)\right] d r=0, \\
\beta_{1}(s) c_{2}(s)+\beta_{2}(s) c_{1}(s)+\int_{a}^{\beta}\left[c_{2}(r) B_{1}(r, s)\right. \\
\left.\quad+c_{1}(r) B_{2}(r, s)\right] d r=0
\end{array}
\end{aligned}
$$

possess a common non-trivial solution $c_{1}(s), c_{2}(s)$. These functions $c_{1}(s)$, $c_{2}(s)$ then satisfy (3); and conversely every pair of functions $c_{1}(s), c_{2}(s)$ which satisfy (3) also satisfy (6).

These theorems follow immediately from the definitions of dependence and independence so that no proof will be needed.

Let us now consider, more in detail, the case in which, for every value of $s$ in $J_{s}$,

$$
\Delta(s) \equiv\left|\begin{array}{ll}
\alpha_{1}(s) & \alpha_{2}(s) \\
\beta_{1}(s) & \beta_{2}(s)
\end{array}\right| \neq 0
$$

Equations (6) may now be reduced to

$$
c_{i}(s)=\int_{a}^{\beta}\left[K_{i 1}(s, t) c_{1}(t)+K_{i 2}(s, t) c_{2}(t)\right] d t \quad(i=1,2),
$$

where

$$
K_{i j}(s, t)=\frac{(-1)^{i}}{\Delta(s)}\left|\begin{array}{ll}
\alpha_{i}(s) & A_{3-j}(t, s) \\
\beta_{i}(s) & B_{3-j}(t, s)
\end{array}\right|
$$


We shall call the set of functions

$$
\begin{array}{ll}
K_{11}(s, t), & K_{12}(s, t), \\
K_{21}(s, t), & K_{22}(s, t)
\end{array}
$$

the kernel-system of (7).

By the side of (7) we consider the associated non-homogeneous systent

(10) $\quad c_{i}(s)=f_{i}(s)+\int_{a}^{\beta}\left[K_{i 1}(s, t) c_{1}(t)+K_{i 2}(s, t) c_{2}(t)\right] d t \quad(i=1,2)$.

We define with Fredholm two new intervals

$$
J_{s}^{(1)}: \quad \alpha_{1} \leqq s \leqq \beta_{1} ; \quad J_{s}^{(2)}: \quad \alpha_{2} \leqq s \leqq \beta_{2},
$$

such that $\alpha_{1}<\beta_{1} \leqq \alpha_{2}<\beta_{2}$,

$$
\beta_{1}-\alpha_{1}=\beta_{2}-\alpha_{2}=\beta-\alpha .
$$

Let $J_{s}^{\prime}$ denote the combined interval of $J_{s}^{(1)}$ and $J_{s}^{(2)}$, so that

$$
\int_{J} F(s) d s=\int_{J(1)} F(s) d s+\int_{J^{(2)}} F(s) d s .
$$

We have also four square regions $J_{s t}^{(i, j)}(i, j=1,2)$ to consider, and $J_{\iota t}^{\prime}$ will be used to indicate the totality of all the squares. Then we will map our functions into the new intervals and regions in such a way that the functions $K_{i j}$ composing the kernel system each occupy one of the four squares. That is, we define

$$
\begin{aligned}
& K(s, t)=K_{i j}\left(s-\alpha_{i}+\alpha, t-\alpha_{j}+\alpha\right) \quad \text { for } \quad J_{s t}^{i j}, \\
& f(s)=f_{i .}\left(s-\alpha_{i}+\alpha\right) \quad \text { for } J_{i}^{i}, \\
& c(s)=c_{i}\left(s-\alpha_{i}+\alpha\right) \quad \text { for } J_{s}^{i} \quad(i, j=1,2) .
\end{aligned}
$$

According to this notation, the equations (11) have the form

$$
c(s)=f(s)+\int_{J^{\prime}} K(s, t) c(t) d t .
$$

This equation may be treated as an ordinary Fredholm equation by forming the Fredholm determinant, $\Delta$, and first minor, $\Delta(s, t)$, of the kernel $K(s, t)$ in the usual way. We shall call $\Delta$ the determinant of the kernel-system (9).

If $\Delta \neq 0$, the resolvent function

$$
Q(s, t)=\frac{\Delta(s, t)}{\Delta},
$$


satisfies the relations

$$
\begin{aligned}
& Q(s, t)=K(s, t)+\int_{J^{\prime}} Q(s, \sigma) K(\sigma, t) d \sigma \\
& Q(s, t)=K(s, t)+\int_{J^{\prime}} K(s, \sigma) Q(\sigma, t) d \sigma
\end{aligned}
$$

and the equation (12) has one and only one solution,

$$
c(s)=f(s)+\int_{J} Q(s, t) f(t) d t .
$$

Returning to the old coördinates, we can define

$$
Q_{i j}(s, t)=Q\left(s-\alpha+\alpha_{i}, t-\alpha+\alpha_{j}\right) \quad\left(\text { for } J_{s t}^{i j}\right) .
$$

This is called the resolvent-system of the kernel-system $K_{i j}$. Equations (13) become

$$
\begin{aligned}
& Q_{i j}(s, t)=K_{i j}(s, t)+\int_{\alpha}^{\beta}\left[Q_{i 1}(s, \sigma) K_{1 j}(\sigma, t)\right. \\
& \left.+Q_{i 2}(s, \sigma) K_{2 j}(\sigma, t)\right] d \sigma, \\
& Q_{i j}(s, t)=K_{i j}(s, t)+\int_{a}^{\beta}\left[K_{i 1}(s, \sigma) Q_{1 j}(\sigma, t)\right. \\
& \left.+K_{i 2}(s, \sigma) Q_{2 j}(\sigma, t)\right] d \sigma,
\end{aligned}
$$

and the solution (14) takes the form

$$
c_{i}(s)=f_{i}(s)+\int_{a}^{\beta \beta}\left[Q_{i 1}(s, t) f_{1}(t)+Q_{i 2}(s, t) f_{2}(t)\right] d t \quad(i=1,2) .
$$

We now easily infer the truth of the following lemmas:

LemmA I. A necessary and sufficient condition that the system (10) possess a unique solution is that $\Delta \neq 0$. If this condition is satisfied, the solution is given by formula (16); and, in particular, the trivial solution will be the only solution of the homogeneous system (7).

Lemma II. When $\Delta=0$, the system (7) always possesses non-trivial solutions; and a necessary and sufficient condition that the system (10) have solutions is that the equation

$$
\int_{J} \psi(s) f(s) d s=\int_{\alpha}^{\beta}\left[\psi_{1}(s) f_{1}(s)+\psi_{2}(s) f_{2}(s)\right] d s=0
$$

be satisfied by every solution $\psi(s)$ of the equation

$$
\psi(s)=\int_{J^{\prime}} \psi(t) K(t, s) d t .
$$

By combining Lemma I and Theorem $\mathrm{V}$ we have 
Theorem VI. If $U_{1}, U_{2}$ are self-independent and fulfill the condition (D) for every value of $S$ in $J_{s}$, then a necessary and sufficient condition that they be independent of each other is that the Fredholm determinant $\Delta$ of the kernel-system (9) be different from zero.

Theorem VII. If $U_{1}, U_{2}$ fulfill the condition $(D)$, the equations

$$
U_{1}=\phi_{1}(s), \quad U_{2}=\phi_{2}(s),
$$

when regarded as equations in $u_{1}(s)$ and $u_{2}(s)$, possess a unique solution if and only if $U_{1}, U_{2}$ are independent. This solution is integro-lineqr in $\phi_{1}$ and $\phi_{2}$.

For, if we replace $u_{1}$ and $u_{2}$ by

$$
\begin{aligned}
& v_{1}(s)=\alpha_{2}(s) u_{1}(s)+\beta_{2}(s) u_{2}(s), \\
& v_{2}(s)=\alpha_{1}(s) u_{1}(s)+\beta_{1}(s) u_{2}(s),
\end{aligned}
$$

and let $f_{1}=\phi_{2}, f_{2}=\phi_{1}$, equations (19) become

$$
v_{i}(s)=f_{i}(s)+\int_{a}^{\beta}\left[v_{1}(r) K_{1 i}(r, s)+v_{2}(r) K_{2 i}(r, s)\right] d r \quad(i=1,2),
$$

or simply

$$
v(s)=f(s)+\int_{J^{\prime}} v(r) K(r, s) d r .
$$

This equation however has precisely the transposed kernel $K(r, s)$, so that it has a unique solution when and only when $\Delta \neq 0$. The second part of the theorem now follows readily.

Corollary I. If $(D)$ is fulfilled, the homogeneous equations $U_{1}=0$, $U_{2}=0$ possess non-trivial solutions when and only when $U_{1}, U_{2}$ are dependent.

Corollary II. If $U_{1}$ is such that $\alpha_{1}(s), \beta_{1}(s)$ do not vanish together* and if $U_{1}=0$ admits no non-trivial solution, then every self-independent $U_{2}$ which fulfills $(D)$ is independent of $U_{1}$.

An important application of this corollary is that for a given self-independent $U_{1}$ in which $\alpha_{1}(s), \beta_{1}(s)$ do not vanish together, if there can be found a $U_{2}$ such that $(D)$ is satisfied and for which $U_{2}=0$ admits no solution other than the trivial one, then $U_{1}, U_{2}$ are independent. Unfortunately, I have as yet been unable to determine whether such a $U_{2}$ always exists. We shall have to leave this important general problem without giving a definite answer. Instead we shall only show the following fact which includes several important special cases in which we know $U_{2}$ can be found.

* Obviously $(D)$ cannot be fulfilled if $\alpha_{1}(s), \beta_{1}(s)$ do vanish together. On the other hand, if $\alpha_{1}(s), \beta_{1}(s)$ do not vanish together, there always exist functions $\alpha_{2}(s), \beta_{2}(s)$ such that $(D)$ is fulfilled, for instance $\alpha_{2}=-\beta_{1}, \beta_{2}=\alpha_{1}$. 
THEOREM VIII. If for a given self-independent expression

$$
\begin{aligned}
U_{1}[u] \equiv \alpha_{1}(s) u_{1}(s)+\beta_{1}(s) & u_{2}(s) \\
& +\int_{a}^{\beta}\left[A_{1}(s, r) u_{1}(r)+B_{1}(\varepsilon, r) u_{2}(r)\right] d r,
\end{aligned}
$$

in which $\alpha_{1}(s), \beta_{1}(s)$ do not vanish together, there can be found constants $k_{1}$, $k_{2}$ such that $k_{1} \alpha_{1}(s), k_{2} \beta_{1}(s)$ do not vanish together and such that

$$
\begin{aligned}
U_{1}^{\prime}[u] \equiv k_{1} \alpha_{1}(s) u_{1}(s) & +k_{2} \beta_{1}(s) u_{2}(s) \\
+ & \int_{a}^{\beta}\left[k_{1} A_{1}(s, r) u_{1}(r)+k_{2} B_{1}(s, r) u_{2}(r)\right] d r=0
\end{aligned}
$$

admits no non-trivial solution, then it is possible to find a $U_{2}$ such that $(D)$ is fulfilled and that $U_{1}, U_{2}$ are independent.

For, suppose $k_{1}, k_{2}$ are both different from zero, then the theorem is obvious, because if we group the constants $k_{1}, k_{2}$ with the unknown functions $u_{1}(s)$, $u_{2}(s)$ respectively, then $U_{1}^{\prime}$ will have exactly the same form as $U_{1}$ so that they both can have no solution. By Corollary II, a $U_{2}$ exists.

If $k_{2}=0$, then we must have $k_{1} \neq 0, \alpha_{1}(s) \neq 0$ for every value of $s$ in $J_{8}$, and, further, on dividing $U_{1}^{\prime}$ by $k_{1}$, the equation

$$
U_{1} \equiv \alpha_{1}(s) u_{1}(s)+\int_{\alpha}^{\beta} A_{1}(s, r) u_{1}(r) d r=0
$$

has no non-trivial solution $u_{1}(s)$. Now if we define

$$
U_{2} \equiv \alpha_{1}(s) u_{2}(s)+\int_{\alpha}^{\beta} A_{1}(s, r) u_{2}(r) d r,
$$

then $U_{2}=0$ will have no non-trivial solution $u_{2}(s)$, so that the equations $U_{1}=0, U_{2}=0$ together will admit only the trivial solution $u_{1}(s)=0$, $u_{2}(s)=0$. The same argument will enable us to construct a $U_{2}$ for the case $k_{1}=0$.

\section{The Adjoint Integro-Differential Expression}

Definition. The integro-differential expressions

$$
\begin{gathered}
L[u] \equiv \frac{\partial u(x, s)}{\partial x}+\phi(x, s) u(x, s)+\int_{a}^{\beta} \psi\left(\begin{array}{r}
s \\
x t
\end{array}\right) u(x, t) d t, \\
M[v] \equiv-\frac{\partial v(x, s)}{\partial x}+\phi(x, s) v(x, s)+\int_{a}^{\beta} \psi\left(\begin{array}{r}
t \\
x s
\end{array}\right) v(x, t) d t
\end{gathered}
$$


are said to be adjoint to each other; the equations

$$
\begin{array}{ll}
\left(A_{0}\right) & L[u]=0, \\
\left(\bar{A}_{0}\right) & M[v]=0
\end{array}
$$

are called adjoint equations.

If we multiply $L[u], M[v]$ respectively by $v(x, s)$ and $u(x, s)$, integrate with respect to $s$, and subtract the results, we find

$$
\text { (1) } \int_{a}^{\beta}[v(x, s) L[u]-u(x, s) M[v]] d s=\frac{\partial}{\partial x} \int_{a}^{\beta} u(x, s) v(x, s) d s \text {, }
$$

which may be called Lagrange's Identity. Integrating again, with respect to $x$, we have the Green's theorem:

$$
\begin{aligned}
\int_{x_{1}}^{x_{2}} \int_{a}^{\beta}[v L[u]-u M[v]] d s d x & \\
& =\int_{a}^{\beta}\left[u\left(x_{2}, s\right) v\left(x_{2}, s\right)-u\left(x_{1}, s\right) v\left(x_{1}, s\right)\right] d s .
\end{aligned}
$$

These relations hold for any continuous functions $u(x, s)$ and $v(x s)$, provided they have continuous first derivatives with respect to $x$.

Let us write for convenience $\left(\bar{A}_{0}\right)$ in the form

$$
\left(\bar{A}_{0}^{\prime}\right)-M[v] \equiv \frac{\partial v(x, s)}{\partial x}+\bar{\phi}(x, s) v(x, s)+\int_{a}^{\beta} \bar{\psi}\left(\begin{array}{c}
s \\
x t
\end{array}\right) v(x, t) d t=0
$$

A dash above a function will be used here consistently to indicate the corresponding function of the adjoint equation. The solution of $\left(\bar{A}_{0}^{\prime}\right)$ may then be written

$$
v(x, s)=\bar{R}\left(\begin{array}{l}
x s \\
y
\end{array}\right) v(y, s)+\int_{a}^{\beta} \bar{S}\left(\begin{array}{l}
x s \\
y t
\end{array}\right) v(y, t) d t
$$

There are important symmetrical relations between the functions

$$
R\left(\begin{array}{l}
x s \\
y
\end{array}\right), S\left(\begin{array}{l}
x s \\
y t
\end{array}\right), \bar{R}\left(\begin{array}{l}
x s \\
y
\end{array}\right), \text { and } \bar{S}\left(\begin{array}{l}
x s \\
y t
\end{array}\right)
$$

To obtain such relations, let us apply Green's theorem to the solutions of $\left(A_{0}\right)$ and $\left(\bar{A}_{0}^{\prime}\right)$. For such functions, $u, v$, Green's theorem becomes

$$
\int_{\alpha}^{\beta} u\left(x_{1}, s\right) v\left(x_{1}, s\right) d s=\int_{\alpha}^{\beta} u\left(x_{2}, s\right) v\left(x_{2}, s\right) d s
$$

for any pair of values $x_{1}, x_{2}$ in $I_{x}$.

Let $x_{3}, x_{4}$ be respectively the points at which the initial functions of $u, v$ 
are to be assigned. Then, by (12), $\S 2$, the solutions have the forms

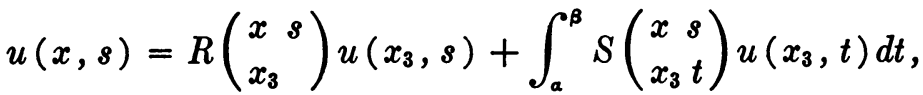

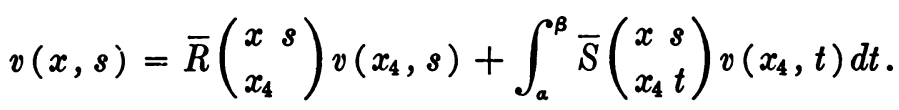

Substituting into (4) and regrouping the terms, we find

where

$$
\int_{a}^{\beta} f(s) u\left(x_{3}, s\right) d s=0,
$$

$$
\begin{aligned}
f(s)=\left[R\left(\begin{array}{l}
x_{1} s \\
x_{3}
\end{array}\right) \bar{R}\left(\begin{array}{l}
x_{1} s \\
x_{4}
\end{array}\right)-R\left(\begin{array}{l}
x_{2} s \\
x_{3}
\end{array}\right) \bar{R}\left(\begin{array}{l}
x_{2} s \\
x_{4}
\end{array}\right)\right] v\left(x_{4}, s\right) \\
\quad+\int_{a}^{\beta}\left[R\left(\begin{array}{l}
x_{1} s \\
x_{3}
\end{array}\right) \bar{S}\left(\begin{array}{l}
x_{1} s \\
x_{4} t
\end{array}\right)+\bar{R}\left(\begin{array}{l}
x_{1} t \\
x_{4}
\end{array}\right) S\left(\begin{array}{l}
x_{1} t \\
x_{3} s
\end{array}\right)\right. \\
\quad+\int_{a}^{\beta} S\left(\begin{array}{l}
x_{1} r \\
x_{3} s
\end{array}\right) \bar{S}\left(\begin{array}{l}
x_{1} r \\
x_{4} t
\end{array}\right) d r-R\left(\begin{array}{l}
x_{2} s \\
x_{3}
\end{array}\right) \bar{S}\left(\begin{array}{l}
x_{2} s \\
x_{4} t
\end{array}\right) \\
\left.-\bar{R}\left(\begin{array}{l}
x_{2} t \\
x_{4}
\end{array}\right) S\left(\begin{array}{l}
x_{2} t \\
x_{3} s
\end{array}\right)-\int_{a}^{\beta} S\left(\begin{array}{l}
x_{2} r \\
x_{3} s
\end{array}\right) \bar{S}\left(\begin{array}{l}
x_{2} r \\
x_{4} t
\end{array}\right) d r\right] v\left(x_{4}, t\right) d t .
\end{aligned}
$$

Since the initial function $u\left(x_{3}, s\right)$ is arbitrary, we conclude that $f(s) \equiv 0$. Moreover, the initial function $v\left(x_{4}, s\right)$ is also arbitrary, so that, by the lemma to be proved presently, we obtain the following identities:

$$
\begin{array}{r}
R\left(\begin{array}{l}
x_{1} s \\
x_{3}
\end{array}\right) \bar{R}\left(\begin{array}{l}
x_{1} s \\
x_{4}
\end{array}\right)=R\left(\begin{array}{l}
x_{2} s \\
x_{3}
\end{array}\right) \bar{R}\left(\begin{array}{l}
x_{2} s \\
x_{4}
\end{array}\right), \\
R\left(\begin{array}{l}
x_{1} s \\
x_{3}
\end{array}\right) \bar{S}\left(\begin{array}{l}
x_{1} s \\
x_{4} t
\end{array}\right)+\bar{R}\left(\begin{array}{l}
x_{1} t \\
x_{4}
\end{array}\right) S\left(\begin{array}{l}
x_{1} t \\
x_{3} s
\end{array}\right)+\int_{a}^{\beta} S\left(\begin{array}{l}
x_{1} r \\
x_{3} s
\end{array}\right) \bar{S}\left(\begin{array}{l}
x_{1} r \\
x_{4} t
\end{array}\right) d r \\
=R\left(\begin{array}{l}
x_{2} s \\
x_{3}
\end{array}\right) \bar{S}\left(\begin{array}{l}
x_{2} s \\
x_{4} t
\end{array}\right)+\bar{R}\left(\begin{array}{l}
x_{2} t \\
x_{4}
\end{array}\right) S\left(\begin{array}{l}
x_{2} t \\
x_{3} s
\end{array}\right) \\
+\int_{a}^{\beta} S\left(\begin{array}{l}
x_{2} r \\
x_{3} s
\end{array}\right) \bar{S}\left(\begin{array}{l}
x_{2} r \\
x_{4} t
\end{array}\right) d r .
\end{array}
$$

These relations hold identically in $I_{x_{1} x_{2} x_{3} x_{4}} J_{s}$ and $I_{x_{1} x_{2} x_{3} x_{4}} J_{s t}$ respectively. In particular, if we let $x=x_{1}=x_{3}, y=x_{2}=x_{4}$, we have*

$$
\bar{R}\left(\begin{array}{l}
x s \\
y
\end{array}\right)=R\left(\begin{array}{l}
y s \\
x
\end{array}\right), \quad \bar{S}\left(\begin{array}{l}
x s \\
y t
\end{array}\right)=S\left(\begin{array}{l}
y t \\
x s
\end{array}\right) .
$$

* The first relation (6), and also the first relation (7), may also be inferred from the definition of $R$; see (2), $\$ 2$. 
Letting $x=x_{1}, y=x_{2}=x_{3}=x_{4}$, we have

$$
\begin{gathered}
R\left(\begin{array}{l}
x s \\
y
\end{array}\right) \bar{R}\left(\begin{array}{l}
x s \\
y
\end{array}\right)=1, \\
R\left(\begin{array}{l}
x s \\
y
\end{array}\right) \bar{S}\left(\begin{array}{l}
x s \\
y t
\end{array}\right)+\bar{R}\left(\begin{array}{l}
x t \\
y
\end{array}\right) S\left(\begin{array}{l}
x t \\
y s
\end{array}\right)+\int_{a}^{\beta} S\left(\begin{array}{l}
x r \\
y s
\end{array}\right) \bar{S}\left(\begin{array}{l}
x r \\
y t
\end{array}\right) d r=0 .
\end{gathered}
$$

A special case of interest is when $L[u]$ is anti-self-adjoint, i. e., when $L[u] \equiv-M[u]$. In this case, we must have

$$
\phi(x, s) \equiv 0, \quad \psi\left(\begin{array}{r}
s \\
x t
\end{array}\right) \equiv-\psi\left(\begin{array}{r}
t \\
x s
\end{array}\right) .
$$

Consequently, we have

$$
R\left(\begin{array}{l}
x s \\
y
\end{array}\right) \equiv 1, \quad S\left(\begin{array}{l}
x s \\
y t
\end{array}\right) \equiv \bar{S}\left(\begin{array}{l}
x s \\
y t
\end{array}\right)=S\left(\begin{array}{l}
y t \\
x s
\end{array}\right) .
$$

We will now prove the lemma which we have referred to, and which will be useful again later.

Lemma. If $h(s)$ and $H(s, t)$ are continuous functions such that

$$
h(s) \phi(s)+\int_{a}^{\beta} H(s, t) \phi(t) d t \equiv 0
$$

for every continuous function $\phi(s)$, then $h(s) \equiv 0$ and $H(s, t) \equiv 0$.

It is sufficient to show $h\left(s_{0}\right)=0$ when $\alpha<s_{0}<\beta$, because it will then follow from the continuity of $h$ that $h(s) \equiv 0$, and therefore $H(s t) \equiv 0$.

Let $s_{0}$ be any interior point of the interval $J_{s}$. Let a particular function $\phi(s)$ be defined as follows:

$$
\phi(s)= \begin{cases}0 & \text { for } \quad\left|s-s_{0}\right|>\epsilon, \\ 1 & \text { for } \quad s=s_{0}, \\ \text { continuous, positive and } \leqq 1 & \text { for } \quad\left|s-s_{0}\right| \leqq \epsilon .\end{cases}
$$

Then from (10)

$$
h\left(s_{0}\right)+\int_{s_{0}-\epsilon}^{s_{0}+e} H\left(s_{0}, t\right) \phi(t) d t=0 .
$$

By the first law of the mean,

$$
h\left(s_{0}\right)+2 \epsilon H\left(s_{0}, t_{1}\right) \phi\left(t_{1}\right)=0,
$$

where $s_{0}-\epsilon<t_{1}<s_{0}+\epsilon$. Let $|H(s, t)|<M$, then

$$
\left|h\left(s_{0}\right)\right|=2 \epsilon \phi\left(t_{1}\right)\left|H\left(s_{0}, t_{1}\right)\right|<2 \epsilon M ;
$$

hence $h\left(s_{0}\right)=0$. 


\section{A Modified Form for Green's Theorem*}

Let $U_{1}[u ; s], U_{2}[u ; s]$ be the two integro-linear forms

$$
\begin{aligned}
U_{i}[u ; s] \equiv & \alpha_{i}(s) u(a, s)+\beta_{i}(s) u(b, s) \\
& +\int_{a}^{\beta}\left[A_{i}(s, r) u(a, r)+B_{i}(s, r) u(b, r)\right] d r \quad(i=1,2) .
\end{aligned}
$$

Regarding (1) as equations in $u(a, s)$ and $u(b, s)$, and $U_{1}[u ; s], U_{2}[u ; s]$ as known functions of $s$, it is seen (Theorem VII, $\S 4$ ) that if the condition $(D)$ is fulfilled, it is possible to solve for $u(a, s), u(b, s)$ uniquely in terms of $U_{1}$ and $U_{2}$, provided the forms are independent; and furthermore, that the unique solution will consist of two integro-linear forms in $U_{1}$ and $U_{2}$ of the same form as (1). In this case, the second member of Green's Theorem $((2), \S 5)$, in which we put $x_{1}=a$ and $x_{2}=b$, thus becomes

$$
\begin{aligned}
\int_{a}^{\beta}[u(b, s) v(b, s) & -u(a, s) v(a, s)] d s \\
= & \int_{a}^{\beta}\left(U_{1}[u ; s] V_{2}[v ; s]+U_{2}[u ; s] V_{1}[v ; s]\right) d s,
\end{aligned}
$$

where $V_{1}[v ; s], V_{2}[v ; s]$ are integro-linear forms in $v(a, s)$ and $v(b, s)$ of the form

$$
\begin{aligned}
V_{i}[v ; s] \equiv & \gamma_{i}(s) v(a, s)+\delta_{i}(s) v(b, s) \\
& +\int_{a}^{\beta}\left[C_{i}(s, r) v(a, r)+D_{i}(s, r) v(b, r)\right] d r \quad(i=1,2) .
\end{aligned}
$$

Thus we see that Green's theorem may always be written in the form

$$
\begin{aligned}
\int_{a}^{b} \int_{a}^{\beta}(v(x, s) L[u] & -u(x, s) M[v] d s d x) \\
= & \int_{a}^{\beta}\left(U_{1}[u ; s] V_{2}[v ; s]+U_{2}[u ; s] V_{1}[v ; s]\right) d s
\end{aligned}
$$

if $U_{1}$ and $U_{2}$ are independent and satisfy condition $(D)$.

Now, suppose $(D)$ is not satisfied, or that $(D)$ is satisfied but $U_{1}$ and $U_{2}$ are dependent. Will it be still possible to determine $V_{1}$ and $V_{2}$ so that the identity (2) will hold? Let us find the conditions under which $V_{1}, V_{2}$ can be determined so as to satisfy (2).

Assuming that $U_{1}, U_{2}$ have the form (1) and $V_{1}, V_{2}$ the form (3), let us then determine the continuous functions $\gamma_{i}, \delta_{i}, C_{i}, D_{i}(i=1,2)$ so that

*In connection with $\S \S 6,7$ see the corresponding developments for differential equations given in these Tra, s a ctions by Birkhoff, vol. 9 (1908), p. 373, and Bôcher, vol. 14 (1913), p. 415. 
(2) holds for every set of continuous functions $u(a, s), u(b, s), v(a, s)$, $v(b, s)$. It may be remarked here that the notations $U_{i}[u ; s], U_{i}[u]$, $U_{i}(s), U_{i}$ will be used indiscriminately for convenience, the same being true for $V_{i}$.

On substituting (1) in (2) and equating the coefficients of the arbitrary functions $u(a, s)$ and $u(b, s)$, we obtain

$$
\begin{aligned}
\alpha_{2}(s) V_{1}[v ; s]+\alpha_{1}(s) V_{2}[v ; s] & +\int_{a}^{\beta}\left(V_{1}[v ; r] A_{2}(r, s)\right. \\
& \left.+V_{2}[v ; r] A_{1}(r, s)\right) d r=-v(a, s),
\end{aligned}
$$

$$
\begin{aligned}
\beta_{2}(s) V_{1}[v ; s]+\beta_{1}(s) V_{2}[v ; s]+ & \int_{a}^{\beta}\left(V_{1}[v ; r] B_{2}(r, s)\right. \\
& \left.+V_{2}[v ; r] B_{1}(r, s)\right) d r=v(b, s)
\end{aligned}
$$

as a necessary and sufficient condition that $U_{1}, U_{2}$ defined by (1) should satisfy (2). Substituting in these equations the expressions for $V_{1}$ and $V_{2}$ from (3) and collecting the coefficients of the arbitrary functions $v(a, s)$ and $v(b, s)$, we find, by the lemma proved at the end of $\S 5$, that the following identities give a necessary and sufficient condition for $V_{1}, V_{2}$ as defined by (3) to satisfy (5):

$$
\begin{gathered}
\alpha_{2}(s) \gamma_{1}(s)+\alpha_{1}(s) \gamma_{2}(s)+1=0, \\
\beta_{2}(s) \gamma_{1}(s)+\beta_{1}(s) \gamma_{2}(s)=0, \\
\alpha_{2}(s) \delta_{1}(s)+\alpha_{1}(s) \delta_{2}(s)=0, \\
\beta_{2}(s) \delta_{1}(s)+\beta_{1}(s) \delta_{2}(s)-1=0, \\
\alpha_{2}(s) C_{1}(s, r)+\alpha_{1}(s) C_{2}(s, r)+\gamma_{1}(r) A_{2}(r, s) \\
+\gamma_{2}(r) A_{1}(r, s) \\
+\int_{a}^{\beta}\left(A_{2}(t, s) C_{1}(t, r)+A_{1}(t, s) C_{2}(t, r)\right) d t=0, \\
\beta_{2}(s) C_{1}(s, r)+\beta_{1}(s) C_{2}(s, r)+\gamma_{1}(r) B_{2}(r, s) \\
+\gamma_{2}(r) B_{1}(r, s) \\
+\int_{a}^{\beta}\left(B_{2}(t, s) C_{1}(t, r)+B_{1}(t, s) C_{2}(t, r)\right) d t=0,
\end{gathered}
$$

(7a) 


$$
\begin{aligned}
\alpha_{2}(s) D_{1}(s, r) & +\alpha_{1}(s) D_{2}(s, r)+\delta_{1}(r) A_{2}(r, s) \\
& +\delta_{2}(r) A_{1}(r, s) \\
& +\int_{a}^{\beta}\left(A_{2}(t, s) D_{1}(t, r)+A_{1}(t, s) D_{2}(t, r)\right) d t=0, \\
\beta_{2}(s) D_{1}(s, r) & +\beta_{1}(s) D_{2}(s, r)+\delta_{1}(r) B_{2}(r, s) \\
& +\delta_{2}(r) B_{1}(r, s) \\
& +\int_{a}^{\beta}\left(B_{2}(t, s) D_{1}(t, r)+B_{1}(t, s) D_{2}(t, r)\right) d t=0 .
\end{aligned}
$$

Thus these eight equations form a necessary and sufficient condition that $U_{1}$, $U_{2}, V_{1}, V_{2}$ as defined by (1) and (3) satisfy (2). We will now inquire under what conditions the continuous functions $\gamma_{i}, \delta_{i}, C_{i}, D_{i}(i=1,2)$ can be determined to satisfy equations $(6 a),(6 b),(7 a),(7 b)$.

If $\Delta(s) \neq 0$ for every value of $s$ in $J_{s}$, there will be a unique solution of equations $(6 a)$ and $(6 b)$, namely

$$
\gamma_{i}(s)=(-1)^{i-1} \frac{\beta_{i}(s)}{\Delta(s)}, \quad \delta_{i}(s)=(-1)^{i-1} \frac{\alpha_{i}(s)}{\Delta(s)} \quad(i=1,2) .
$$

On the other hand, if for a particular value, $s_{0}$, we have $\Delta\left(s_{0}\right)=0$, then, in order that the matrix and the augmented matrix of the system (6a) have the same rank, we must have $\beta_{1}\left(s_{0}\right)=\beta_{2}\left(s_{0}\right)=0$. But this cannot be the case, as we see from the second equation (6b). Consequently, $\gamma_{i}, \delta_{i}$ cannot be determined when the condition $(D)$ is not fulfilled. The condition $(D)$ is then a first necessary condition that we have to impose on $U_{1}, U_{2}$ in order that the problem in question be possible.

Assuming then that $(D)$ is satisfied by $U_{1}$ and $U_{2}$, let us now consider the system $(7 a)$.

Using the notation (8), $\S 4$ and letting

$$
\begin{aligned}
& f_{i}(s, r)=\left|\begin{array}{rr}
\gamma_{1}(r) & K_{i 2}(s, r) \\
-\gamma_{2}(r) & K_{i 1}(s, r)
\end{array}\right|, \\
& g_{i}(s, r)=\left|\begin{array}{rr}
\delta_{1}(r) & K_{i 2}(s, r) \\
-\delta_{2}(r) & K_{i 1}(s, r)
\end{array}\right|,
\end{aligned}
$$

the equations ( $7 a$ ) may readily be reduced to the form

$$
\begin{aligned}
C_{i}(s, r)=f_{i}(s, r)+\int_{a}^{\beta}\left[K_{i 1}(s, t) C_{1}(t, r)\right. \\
\left.\quad+K_{i 2}(s, t) C_{2}(t, r)\right] d t \quad(i=1,2) .
\end{aligned}
$$

In this form we have precisely a system of equations of the type (10), $\S 4$. 
Now if the Fredholm determinant $\Delta$ of the kernel system $K_{i j}(s, t),(i, j=1$, 2 ), is different from zero, we have by Lemma I, $\$ 4$, a unique solution of the equations, which is given by

$$
C_{i}(s, r)=f_{i}(s, r)+\int_{a}^{\beta}\left[Q_{i 1}(s, t) f_{1}(t, r)+Q_{i 2}(s, t) f_{2}(t, r)\right] d t
$$

Because of (9) and the resolvent relations (15), $\S 4$, this solution simplifies into

$$
C_{i}(s, r)=\left|\begin{array}{rr}
\gamma_{1}(r) & Q_{i 2}(s, r) \\
-\gamma_{2}(r) & Q_{i 1}(s, r)
\end{array}\right|
$$

and because of (8) it further reduces to

$$
C_{i}(s, r)=\frac{1}{\Delta(r)}\left|\begin{array}{ll}
\beta_{1}(r) & Q_{i 2}(s, r) \\
\beta_{2}(r) & Q_{i 1}(s, r)
\end{array}\right| \quad(i=1,2) .
$$

Similarly, for the system $(7 b)$ we have the unique solution

$$
D_{i}(s, r)=\frac{1}{\Delta(r)}\left|\begin{array}{ll}
\alpha_{1}(r) & Q_{i 2}(s, r) \\
\alpha_{2}(r) & Q_{i 1}(s, r)
\end{array}\right|
$$

On the other hand, if $\Delta=0$, solutions of $(7 a),(7 b)$ both exist by Lemma II, $\S 4$, if and only if

$$
\begin{aligned}
& \int_{a}^{\beta}\left[\psi_{1}(s) f_{1}(s, r)+\psi_{2}(s) f_{2}(s, r)\right] d s=0, \\
& \int_{a}^{\beta}\left[\psi_{1}(s) g_{1}(s, r)+\psi_{2}(s) g_{2}(s, r)\right] d s=0
\end{aligned}
$$

for every non-trivial solution, $\psi_{1}(s), \psi_{2}(s)$, of the equations

$$
\psi_{i}(s)=\int_{a}^{\beta}\left[\psi_{1}(t) K_{1 i}(t, s)+\psi_{2}(t) K_{2 i}(t, s)\right] d t \quad(i=1,2) .
$$

Suppose both of these conditions are satisfied. Substituting the values of $f_{1}, f_{2}, g_{1}, g_{2}$ from (9) and $\gamma_{1}, \gamma_{2}, \delta_{1}, \delta_{2}$ from (8), we have

$$
\begin{aligned}
& \frac{\beta_{1}(r)}{\Delta(r)} \int_{a}^{\beta}\left[\psi_{1}(s) K_{11}(s, r)+\psi_{2}(s) K_{21}(s, r)\right] d s \\
& -\frac{\beta_{2}(r)}{\Delta(r)} \int_{\alpha}^{\beta}\left[\psi_{1}(s) K_{12}(s, r)+\psi_{2}(s) K_{22}(s, r)\right] d s=0, \\
& \frac{\alpha_{1}(r)}{\Delta(r)} \int_{a}^{\beta}\left[\psi_{1}(s) K_{11}(s, r)+\psi_{2}(s) K_{21}(s, r)\right] d s \\
& -\frac{\alpha_{2}(r)}{\Delta(r)} \int_{a}^{\beta}\left[\psi_{1}(s) K_{12}(s, r)+\psi_{2}(s) K_{22}(s, r)\right] d s=0 .
\end{aligned}
$$


These equations may now be regarded as a system of linear algebraic equations whose determinant, $\Delta(r)$, does not vanish for any value of $r$ in $J_{r}$. Whence

$$
\int_{a}^{\beta}\left[\psi_{1}(s) K_{1 i}(s, r)+\psi_{2}(s) K_{2 i}(s, r)\right] d s=0 \quad(i=1,2),
$$

i. e., $\psi_{1} \equiv 0, \psi_{2} \equiv 0$ because of (13). But this is contrary to the fact that $\psi_{1}(s), \psi_{2}(s)$ are a non-trivial solution of (13). Hence $C_{i}, D_{i}$ cannot be determined when $\Delta=0$. Thus we have $\Delta \neq 0$ as a second necessary condition to be imposed on $U_{1}, U_{2}$; that is ( $\S 4$, Theorem VI), $U_{1}, U_{2}$ must be independent in addition to fulfilling the condition $(D)$. Hence

Theorem I. A necessary and sufficient condition that the expressions $V_{1}, V_{2}$ of the type (3) be determinable so that the identity (2) holds for every set of continuous functions $u(a, s), u(b, s), v(a, s), v(b, s)$, is that $U_{1}, U_{2}$ fulfill condition $(D)$ and that they be independent. The determination is unique and given by formulas (3), (8), (11), (12).

Now let us suppose that we start from the expressions $V_{1}, V_{2}$ just determined and that we try to determine $U_{1}, U_{2}$ so as to satisfy (3). We form the determinants $\bar{\Delta}(s), \bar{\Delta}$ for the expressions $V_{1}, V_{2}$ corresponding to the determinants $\Delta(s), \Delta$ for $U_{1}, U_{2}$, and denote by $(\bar{D})$ the condition that, for every value of $s$ in $J_{s}, \bar{\Delta}(s) \neq 0$. Then, by the theorem just stated, since $U_{1}, U_{2}$ do exist, we have the

CoRollary I. If $U_{1}, U_{2}$ are independent and fulfill the condition $(D)$, then the expressions $V_{1}, V_{2}$ are also independent and fulfill the condition $(\bar{D})$. Thus in this case the two sets of expressions are uniquely determinable from each other.

We see that the necessary and sufficient condition of Theorem $\mathrm{I}$ is precisely a necessary and sufficient condition that the system $U_{1}=0, U_{2}=0$, admit no non-trivial solution (Theorem VII, Corollary I, § 4). Hence

Corollary II. If $U_{1}, U_{2}$ are such that the system $U_{1}=0, U_{2}=0$, admits no non-trivial solution, then $V_{1}, V_{2}$ can be determined and they are such that the system $V_{1}=0, V_{2}=0$, admits.no non-trivial solution.

The following fact will be useful later.

Corollary III. If $V_{1}, V_{2}$ exist, then $u(a, s), u(b, s)$ can be uniquely expressed in terms of $U_{1}$ and $U_{2}$ in the form

$$
\begin{aligned}
u(a, s)=-\gamma_{2}(s) U_{1}(s) & -\gamma_{1}(s) U_{2}(s) \\
& -\int_{\alpha}^{\beta}\left[U_{1}(r) C_{2}(r, s)+U_{2}(r) C_{1}(r, s)\right] d r, \\
u(b, s)=\delta_{2}(s) U_{1}(s) & +\delta_{1}(s) U_{2}(s) \\
& +\int_{\alpha}^{\beta}\left[U_{1}(r) D_{2}(r, s)+U_{2}(r) D_{1}(r, s)\right] d r .
\end{aligned}
$$


The existence of a unique solution follows from Theorem VII, $\S 4$, and there it is also shown that the solution is integro-linear in $U_{1}$ and $U_{2}$. Thus we need now only to verify the formulæ (14). For this purpose we assume $u(a, s)=\alpha_{1}^{\prime}(s) U_{1}(s)+\alpha_{2}^{\prime}(s) U_{2}(s)$

$$
+\int_{a}^{\beta}\left[A_{1}^{\prime}(s ., r) U_{1}(r)+A_{2}^{\prime}(s, r) U_{2}(r)\right] d r,
$$

$u(b, s)=\beta_{1}^{\prime}(s) U_{1}(s)+\beta_{2}^{\prime}(s) U_{2}(s)$

$$
+\int_{a}^{\beta}\left[B_{1}^{\prime}(s, r) U_{1}(r)+B_{2}^{\prime}(s, r) U_{2}(r)\right] d r .
$$

Substituting in the expression

$$
\int_{a}^{\triangleright \beta}[u(b, s) v(b, s)-u(a, s) v(a, s)] d s
$$

and collecting the coefficients of $U_{1}$ and $U_{2}$, we find

$$
\begin{aligned}
\int_{a}^{\beta}[u(b, s) v(b, s)-u(a, s) & v(a, s)] d s \\
& =\int_{a}^{\beta}\left[U_{1}(s) V_{2}^{\prime}(s)+U_{2}(s) V_{1}^{\prime}(s)\right] d s,
\end{aligned}
$$

where

$$
\begin{aligned}
V_{i}^{\prime}(s)=-\alpha_{i}^{\prime}(s) v & (a, s)+\beta_{i}^{\prime}(s) v(b, s) \\
& \quad-\int_{a}^{\beta}\left[v(a, r) A_{i}^{\prime}(r, s)-v(b, r) B_{i}^{\prime}(r, s)\right] d r .
\end{aligned}
$$

But (15) is exactly the identity (2). Since we have seen that for each given set of $U_{1}$ and $U_{2}$, the expressions $V_{1}, V_{2}$ are uniquely determined and are given by (3), the expressions (16) and (13) are identical. Hence we have the formulas (14).

\section{The Adjoint System}

It has been seen that the expressions $V_{1}, V_{2}$ are uniquely determined for each $U_{2}$ integro-linearly independent of $U_{1}$ and fulfilling $(D)$. Now let $U_{2}^{\prime}$ be another expression independent of $U_{1}$ and let $V_{1}^{\prime}, V_{2}^{\prime}$ be the corresponding expressions thereby determined. We are to see how the two sets, of $V_{i}$ are related to one another.

The two sets of expressions, $U_{1}, U_{2}, V_{1}, V_{2}$, and $U_{1}, U_{2}^{\prime}, V_{1}^{\prime}, V_{2}^{\prime}$, satisfy the identity (2) of $\S 6$. Consequently,

$$
\begin{aligned}
\int_{a}^{\beta}\left(\left(U_{1}[u ; s] V_{2}[v ; s]\right.\right. & \left.+U_{2}[u ; s] V_{1}[v ; s]\right) d s \\
& =\int_{a}^{\beta}\left(U_{1}[u ; s] V_{2}^{\prime}[v ; s]+U_{2}^{\prime}[u ; s] V_{1}^{\prime}[v ; s]\right) d s .
\end{aligned}
$$


Let $U_{1}, U_{2}, U_{2}^{\prime}$ be written in their full form and the coefficients of the arbitrary functions $u(a, s), u(b, s)$ be equated to zero; we obtain

$$
\begin{aligned}
\alpha_{1}(s) V_{2}[v ; s]+ & \alpha_{2}(s) V_{1}[v ; s] \\
& +\int_{a}^{\beta}\left(A_{1}(r, s) V_{2}[v ; r]+A_{2}(r, s) V_{1}[v ; r]\right) d r \\
= & \alpha_{1}(s) V_{2}^{\prime}[v ; s]+\alpha_{2}^{\prime}(s) V_{1}^{\prime}[v ; s] \\
& \quad+\int_{a}^{\beta}\left(A_{1}(r, s) V_{2}^{\prime}[v ; r]+A_{2}^{\prime}(r, s) V_{1}^{\prime}[v ; r]\right) d r,
\end{aligned}
$$

$$
\begin{aligned}
\beta_{1}(s) V_{2}[v ; s]+ & \beta_{2}(s) V_{1}[v ; s] \\
& +\int_{a}^{\beta}\left(B_{1}(r, s) V_{2}[v ; r]+B_{2}(r, s) V_{1}[v ; r]\right) d r \\
= & \beta_{1}(s) V_{2}^{\prime}[v ; s]+\beta_{2}^{\prime}(s) V_{1}^{\prime}[v ; s] \\
& +\int_{a}^{\beta}\left(B_{1}(r, s) V_{2}^{\prime}[v ; r]_{2}^{\prime}+B_{2}^{\prime}(r, s) V_{1}^{\prime}[v ; r]\right) d r .
\end{aligned}
$$

Let us denote by $\Phi\left[V^{\prime} ; s\right], \Psi\left[V^{\prime} ; s\right]$ respectively the expressions on the right of these equations. Since $\Delta(s) \neq 0$, we find

$$
V_{i}[v ; s]=F_{i}\left[V^{\prime} ; s\right]+\int_{a}^{\beta}\left(K_{i 1}(s, r) V_{1}[v ; r]+K_{i 2}(s, r) V_{2}[v ; r]\right) d r
$$

where

$$
F_{i}\left[V^{\prime} ; s\right]=\frac{(-1)^{i-1}}{\Delta(s)}\left|\begin{array}{cc}
\alpha_{i}(s) & \Phi\left[V^{\prime} ; s\right] \\
\beta_{i}(s) & \Psi\left[V^{\prime} ; s\right]
\end{array}\right| .
$$

The expressions $F_{1}, F_{2}$ are integro-linear and homogeneous in $V_{1}^{\prime}$ and $V_{2}^{\prime}$. As $\Delta \neq 0$, these equations may be solved for $V_{1}$ and $V_{2}$, and the solution is unique, having the form

$$
\begin{array}{r}
V_{i}[v ; s]=F_{i}\left[V^{\prime} ; s\right]+\int_{a}^{\beta}\left(Q_{i 1}(s, r) F_{1}\left[V^{\prime} ; r\right]+Q_{i 2}(s, r) F_{2}\left[V^{\prime} ; r\right]\right) d r \\
(i=1,2) .
\end{array}
$$

The expressions in the second member are obviously integro-linear and homogeneous in $V_{1}^{\prime}$ and $V_{2}^{\prime}$, so that these equations may be regarded as an integrolinear transformation between the expressions $V_{1}, V_{2}$ and $V_{1}^{\prime}, V_{2}^{\prime}$. Upon simplifications due to the resolvent relations (15) of $\S 4$, these equations take the final form

$$
\begin{aligned}
& V_{1}[v ; s]=M_{1}(s) V_{1}^{\prime}[v ; s]+\int_{a}^{\beta} N_{1}(s, r) V_{1}^{\prime}[v ; r] d r, \\
& V_{2}[v ; s]=V_{2}^{\prime}[v ; s]+M_{2}(s) V_{1}^{\prime}[v ; s]+\int_{a}^{\beta} N_{2}(s, r) V_{1}^{\prime}[v ; r] d r .
\end{aligned}
$$


Similar equations may be obtained for expressing $V_{1}^{\prime}, V_{2}^{\prime}$ in terms of $V_{1}, V_{2}$ by solving (1) for $V_{1}^{\prime}, V_{2}^{\prime}$. It is important to notice that both of these integrolinear transformations are unique, since all the coefficients depend only on the coefficients of $U_{1}, U_{2}$, and $U_{2}^{\prime}$.

The importance of the equations (2) lies in the fact that $V_{1}$ is integrolinear and homogeneous in $V_{1}^{\prime}$, so that whenever the boundary condition $V_{1}^{\prime}=0$ is satisfied, the condition $V_{1}=0$ is also satisfied, and vice versa. For this reason, we may state:

Theorem I. The condition $V_{1}=0$ is essentially determined by the condition $U_{1}=0$, and conversely.

Definition. A pair of boundary conditions $U_{1}=0, V_{1}=0$ are said to be adjoint to each other if $U_{1}, V_{1}$ satisfy a relation of the form (2), $\S 6$, where $U_{2}$ is independent of $U_{1}$ and the condition $\Delta(s) \neq 0$ is fulfilled. The systems

$$
\begin{array}{lll}
\left(A_{0}\right) \quad L[u]=0, & \left(B_{0}\right) U_{1}[u]=0, \\
\left(\overline{A_{0}}\right) \quad M[v]=0, & \left(\bar{B}_{0}\right) \quad V_{1}[v]=0
\end{array}
$$

are called adjoint systems.

It follows from Theorem VIII, $\S 4$, that an adjoint boundary condition always exists if the function $\alpha_{1}(s)$ does not vanish in $J_{s}$ and the Fredholm determinant of $\left[-A_{1}(s, r)\right] /\left[\alpha_{1}(s)\right]$ is not zero; and also under certain more general conditions there specified.

As we have done in $\S 3$, we will restrict ourselves to the case in which the system $\left(A_{0}, B_{0}\right)$ is subject to the condition

$$
\alpha_{1}(s)+R\left(\begin{array}{l}
b s \\
a
\end{array}\right) \beta_{1}(s) \neq 0 .
$$

If we consider the adjoint system $\left(\bar{A}_{0}, \bar{B}_{0}\right)$, we find that a similar condition

$$
\gamma_{1}(s)+\bar{R}\left(\begin{array}{l}
b s \\
a
\end{array}\right) \delta_{1}(s) \neq 0
$$

is fulfilled. For, from the formulæ (8), $\S 6$ and (6), (7), $\S 5$, we have

Hence

$$
\begin{aligned}
\gamma_{1}(s)+\bar{R}\left(\begin{array}{l}
b s \\
a
\end{array}\right) \delta_{1}(s) & =\frac{\beta_{1}(s)+R\left(\begin{array}{l}
a s \\
b
\end{array}\right) \alpha_{1}(s)}{\Delta(s)} \\
& =\frac{\alpha_{1}(s)+R\left(\begin{array}{l}
b s \\
a
\end{array}\right) \beta_{1}(s)}{\Delta(s) R\left(\begin{array}{l}
b s \\
a
\end{array}\right)} .
\end{aligned}
$$

ThEOREM II. If the system $\left(A_{0}, B_{0}\right)$ fulfills the condition $(C)$, then the adjoint system $\left(\bar{A}_{0}, \bar{B}_{0}\right)$ fulfills a similar condition $(\bar{C})$. 
We shall next prove

THEOREM III. The adjoint systems $\left(A_{0}, B_{0}\right),\left(\bar{A}_{0}, \bar{B}_{0}\right)$, subject to the condition $(C)$, have the same index.

Let $n$ be the index of the system $\left(A_{0}, B_{0}\right)$ and $m$ that of the adjoint system $\left(\bar{A}_{0}, \bar{B}_{0}\right)$. Let $u_{1}, \cdots, u_{n}$ and $v_{1}, \cdots, v_{m}$ be respectively complete sets of linearly independent solutions of the systems. Let $u$ be any solution of the equation $\left(A_{0}\right)$, and $v$ any solution of $\left(\bar{A}_{0}\right)$.

Applying Green's theorem to $u$ and $v_{i}$, we have

$$
\int_{a}^{\beta} U_{1}[u ; s] V_{2}\left[v_{i} ; s\right] d s=0 \quad(i=1,2, \cdots, m)
$$

for all solutions $u$ of $\left(A_{0}\right)$. As before, let $y$ be any fixed value of $x$ at which the initial function $u(y, s)$ is assigned. Then, by formula (7), $\S 3$,

$$
U_{1}[u ; s]=g(y, s) u(y, s) \div \int_{\alpha}^{\beta} G\left(\begin{array}{r}
s \\
y r
\end{array}\right) u(y, r) d r,
$$

where $g(y, s)$ and $G\left(\begin{array}{c}\dot{y} \\ y\end{array}\right)$ are given by (6), $\S 3$. Because (3) has to hold for all continuous functions $u(y, s)$, we have

or

$$
g(y, s) V_{2}\left[v_{i} ; s\right]+\int_{a}^{\beta} V_{2}\left[v_{i} ; r\right] G\left(\begin{array}{r}
r \\
y s
\end{array}\right) d r=0
$$

$$
g(y, s) V_{2}\left[v_{i} ; s\right]=\int_{a}^{\beta} g(y, r) V_{2}\left[v_{i} ; r\right] K\left(\begin{array}{r}
r \\
y s
\end{array}\right) d r .
$$

That is to say, $\phi_{i}(y, s)=g(y, s) V_{2}\left[v_{i} ; s\right],(i=1,2, \cdots, m)$, are solutions of the equation

$$
\phi(y, s)=\int_{a}^{\beta} \phi(y, r) K\left(\begin{array}{r}
r \\
y s
\end{array}\right) d r .
$$

On the other hand, the initial functions of $u_{1}, \cdots, u_{n}$ form a complete set of linearly independent solutions of the equation

$$
u(y, s)=\int_{\alpha}^{\beta} K\left(\begin{array}{c}
s \\
y r
\end{array}\right) u(y, r) d r
$$

adjoint to (4). Hence if it can be shown (as we will now do) that the functions $V_{2}\left[v_{i} ; s\right](i=1,2, \cdots, m)$ are linearly independent, then $\phi_{i}(y, s)$ will constitute $m$ linearly independent solutions of (4), and therefore $m \leqq n$.

For, suppose $V_{2}\left[v_{i} ; s\right](i=1,2, \cdots, m)$ were linearly dependent. Then there would exist constants $c_{1}, \cdots, c_{m}$, not all zero, such that

Let us define

$$
c_{1} V_{2}\left[v_{1} ; s\right]+\cdots+c_{m} V_{2}\left[v_{m} ; s\right]=0 \text {. }
$$

$$
v_{0}=c_{1} v_{1}+\cdots+c_{m} v_{m} \text {. }
$$


Then $v_{0}$ is also a non-trivial solution of the system $\left(\bar{A}_{0}, \bar{B}_{0}\right)$, and satisfies

$$
V_{2}\left[v_{0} ; s\right]=c_{1} V_{2}\left[v_{1} ; s\right]+\cdots+c_{m} V_{2}\left[v_{m} ; s\right]=0 .
$$

But this is contradictory, because $V_{1}=0, V_{2}=0$ admit no non-trivial solution. This completes the proof that $m \leqq n$.

In the same manner, the functions $\psi_{i}(y, s)=\bar{g}(y, s) U_{2}\left[u_{i} ; s\right](i=1,2$, $\cdots, n)$ form $n$ linearly independent solutions of the equation

$$
\psi(y, s)=\int_{a}^{\beta} \psi(y, r) \bar{K}\left(\begin{array}{r}
r \\
y s
\end{array}\right) d r,
$$

where $\bar{K}$ has the same meaning in the adjoint system $\left(\bar{A}_{0}, \bar{B}_{0}\right)$ as $K$ has in the original system $\left(A_{0}, B_{0}\right)$. On the other hand, the initial functions of $v_{1}, \cdots, v_{m}$ form a complete set of linearly independent solutions of the equation

$$
v(y, s)=\int_{a}^{\beta} \bar{K}\left(\begin{array}{r}
s \\
x y
\end{array}\right) v(y, r) d r
$$

adjoint to (6). Hence $n \leqq m$. When combined with the previous result, we have $m=n$. 'Thus we have established Theorem III and also

Theorem IV. Let $u_{1}, \cdots, u_{n}$ be a complete set of linearly independent solutions of the system $\left(A_{0}, B_{0}\right)$, and $v_{1}, \cdots, v_{n}$ a complete set of linearly independent solutions of the adjoint system $\left(\bar{A}_{0}, \bar{B}_{0}\right)$. Then the functions

$$
\phi_{i}(y, s)=g(y, s) V_{2}\left[v_{i} ; s\right] \quad(i=1,2, \cdots, n)
$$

form a complete set of linearly independent solutions of the equation (4), and the functions

$$
\psi_{i}(y, s)=\bar{g}(y, s) U_{2}\left[u_{i} ; s\right] \quad(i=1,2, \cdots, n)
$$

form a complete set of linearly independent solutions of the equation (6).

If we replace $\phi_{i}(y, s)$ in the equation (10) of $\S 3$ by the values (8), we obtain from the second part of the Corollary of Theorem III, $\S 3$, the

Theorem V. A necessary and sufficient condition that a non-homogeneous system $(A, B)$, subject to the condition $(C)$, possess a solution when the reduced system $\left(A_{0}, B_{0}\right)$ is compatible and when the adjoint system $\left(\bar{A}_{0}, \bar{B}_{0}\right)$ exists, is that

$$
\int_{a}^{\beta} F(y, s) g(y, s) V_{2}\left[v_{i} ; s\right] d s \equiv 0
$$

for every $v_{i}$ which satisfies the adjoint system $\left(\bar{A}_{0}, \bar{B}_{0}\right)$.

By means of (8), $\S 3$, this condition may be given the form

$$
\int_{a}^{\beta}\left(\gamma(s)-U_{1}\left[w\left(\begin{array}{c}
x s \\
y
\end{array}\right)\right]\right) V_{2}\left[v_{i} ; s\right] d s \equiv 0 .
$$




\section{The Self-Adjoint Boundary Conditions}

We have shown that two different choices of the auxiliary boundary expression $U_{2}$ independent of the given $U_{1}$ and fulfilling the condition $(D)$ lead to two expressions $V_{1}$ which are connected by an integro-linear transformation. Furthermore, this transformation is unique in both ways. This fact is important for us here, because in seeking the conditions that a given expression $U_{1}$ be self-adjoint, it is sufficient to seek the conditions that a particular $V_{1}$ thereby determined be connected with $U_{1}$ by an integro-linear transformation.* It is clear that if one particular $V_{1}$ is integro-linearly connected with $U_{1}$, then every $V_{1}$ will be so connected.

Suppose the condition $U_{1}=0$ is self-adjoint, and that, for a particular choice of $U_{2}$, we have

$$
V_{1}[u ; s]=M(s) U_{1}[u ; s]+\int_{a}^{\beta} N(s, t) U_{1}[u ; t] d t,
$$

where

$$
\begin{aligned}
U_{1}[u ; s]=\alpha_{1}(s) u(a, s) & +\beta_{1}(s) u(b, s) \\
& +\int_{a}^{\beta}\left[A_{1}(s, r) u(a, r)+B_{1}(s, r) u(b, r)\right] d r, \\
V_{1}[u ; s]=\gamma_{1}(s) u(a, s) & +\delta_{1}(s) u(b, s) \\
& +\int_{a}^{\beta}\left[C_{1}(s, r) u(a, r)+D_{1}(s, r) u(b, r)\right] d r,
\end{aligned}
$$

the functions $\gamma_{1}, \delta_{1}, C_{1}, D_{1}$ having the values given by the equations (8), (11), and (12) of $\S 6$. The equation (1) may be thrown into the following form

$$
\begin{array}{r}
V_{1}[u ; s]=M(s) \alpha_{1}(s) u(a, s)+M(s) \beta_{1}(s) u(b, s) \\
+\int_{a}^{\beta}\left[M(s) A_{1}(s, r)+N(s, r) \alpha_{1}(r)\right. \\
\left.\quad+\int_{a}^{\beta} N(s, t) A_{1}(t, r) d t\right] u(a, r) d r \\
+\int_{a}^{\beta}\left[M(s) B_{1}(s, r)+N(s, r) \beta_{1}(r)\right. \\
\left.+\int_{a}^{\beta} N(s, t) B_{1}(t, r) d t\right] u(b, r) d r,
\end{array}
$$

whereby we obtain

$$
\gamma_{1}(s)=M(s) \alpha_{1}(s),
$$

* Professor D. Jackson takes the same point of view in his article, in these Tra $\mathrm{n} \mathrm{sac-}$ t i o $\mathrm{s}$, vol. 17 (1916), pp. 418-424. 


$$
\begin{aligned}
& \text { (3) } C_{1}(s, r)=M(s) A_{1}(s, r)+N(s, r) \alpha_{1}(r)+\int_{a}^{\beta} N(s, t) A_{1}(t, r) d t, \\
& \text { (3') } D_{1}(s, r)=M(s) B_{1}(s, r)+N(s, r) \beta_{1}(r)+\int_{a}^{\beta} N(s, t) B_{1}(t, r) d t .
\end{aligned}
$$

Equations (2) and (3) may be regarded as determining the functions $M$ and $N$; equations $\left(2^{\prime}\right)$ and $\left(3^{\prime}\right)$ then constitute the conditions which must be imposed on $U_{1}$ in order that it be self-adjoint.

Substituting the values from (8), $\S 6$, for $\gamma_{1}$ and $\delta_{1}$, the equations (2), $\left(2^{\prime}\right)$ become

$$
\begin{aligned}
& \Delta(s) M(s) \alpha_{1}(s)-\beta_{1}(s)=0 . \\
& \alpha_{1}(s)-\Delta(s) M(s) \beta_{1}(s)=0 .
\end{aligned}
$$

Now $\alpha_{1}(s)$ and $\beta_{1}(s)$ cannot vanish together since we must have $\Delta(s) \neq 0$ throughout $J_{s}$ in order that the adjoint expressions exist. Hence, we must have

$$
\left|\begin{array}{cc}
\Delta(s) M(s) & 1 \\
1 & \Delta(s) M(s)
\end{array}\right|=0
$$

for every value of $s$ in $J$. That is,

It follows that

$$
M(s)= \pm \frac{1}{\Delta(s)} .
$$

$$
\left.\alpha_{1}(s)= \pm \beta_{1}(s) \neq 0 \quad \text { (throughout } J_{s}\right) .
$$

Conversely, when (4) is satisfied,

$$
M(s)= \pm \frac{1}{\Delta(s)} .
$$

Equation (4) is a first necessary condition.

Assuming then that (4) is satisfied, let us proceed to consider the equations (3), $\left(3^{\prime}\right)$. These equations are Fredholm equations with the kernels - $\left[A_{1}(t, r)\right] /\left[\alpha_{1}(r)\right]$ and $-\left[B_{1}(t, r)\right] /\left[\beta_{1}(r)\right]$ respectively. It is conceivable that the Fredholm determinant of either one of these kernels might be zero. We shall now show that in such case, no self-adjoint system is possible.

Let us suppose the Fredholm determinant of the kernel - $\left[A_{1}(t, r)\right]$ / $\left[\alpha_{1}(r)\right]$ to be zero, and a self-adjoint expression to exist so that the equation (3) has a solution. For this it is necessary that

$$
\int_{\alpha}^{\beta} \phi(r)\left[\frac{C_{1}(s, r)}{\alpha_{1}(r)}-\frac{1}{ \pm \Delta(s)} \frac{A_{1}(s, r)}{\alpha_{1}(r)}\right] d r=0
$$


for every non-trivial solution $\phi(r)$ of the transposed equation

$$
\phi(r)=\int_{\alpha}^{\beta}\left[-\frac{A_{1}(r, t)}{\alpha_{1}(t)}\right] \phi(t) d t .
$$

The \pm signs correspond to those of (4). Because of (6), the condition (5) has the form

or, since

$$
\frac{\phi(s)}{ \pm \Delta(s)}+\int_{a}^{\beta} \frac{C_{1}(s, r)}{\alpha_{1}(r)} \phi(r) d r=0
$$

Let us put

$$
\begin{gathered}
\gamma_{1}(s)=\frac{\beta_{1}(s)}{\Delta(s)}=\frac{ \pm \alpha_{1}(s)}{\Delta(s)} \\
\gamma_{1}(s) \frac{\phi(s)}{\alpha_{1}(s)}+\int_{\alpha}^{\beta} C_{1}(s, r) \frac{\phi(r)}{\alpha_{1}(r)} d r=0 .
\end{gathered}
$$

$$
u(a, s)=\frac{\phi(s)}{\alpha_{1}(s)}, \quad u(b, s)=0
$$

Then equations (6) and (7) become

$$
U_{1}[u ; s]=0, \quad V_{1}[u ; s]=0 .
$$

The identity (2), $\S 6$ now becomes, if we let $u(x, s) \equiv v(x, s)$,

$$
-\int_{a}^{\beta}\left[\frac{\phi(s)}{\alpha_{1}(s)}\right]^{2} d s=0,
$$

whence $\phi(s) \equiv 0$. But this is contrary to the fact that $\phi(s)$ is a nontrivial solution of (6). Thus we have derived a second necessary condition for the existence of self-adjoint expressions, namely, that both* $-\left[A_{1}(t, r)\right]$ / $\left[\alpha_{1}(r)\right]$ and $-\left[B_{1}(t, r)\right] /\left[\beta_{1}(r)\right]$ have non-vanishing Fredholm determinants. This condition is sufficient to insure the existence of a unique solution for each of the equations (3), ( $\left.3^{\prime}\right)$, and we shall have a third necessary condition upon equating these solutions to each other. It is also clear that these three necessary conditions combined are also sufficient for the existence of self-adjoint expressions.

To determine the explicit form of the third condition, it is convenient to choose a particular $U_{2}$ which will simplify the computation. We shall choose for instance $U_{2}$ such that

$$
\alpha_{2}(s) \equiv 0, \quad \beta_{2}(s) \equiv 1, \quad A_{2}(s, r) \equiv B_{2}(s, r) \equiv 0 .
$$

This $U_{2}$ is integro-linearly independent of the given $U_{1}$. To prove this we

\footnotetext{
* The proof for $-\left[B_{1}(t, r)\right] /\left[\beta_{1}(r)\right]$ proceeds exactly in the same way.
} 
note that $\Delta(s)=\alpha_{1}(s) \neq 0$ in $J_{s}$; and according to the notations of $\S 4$,

$$
\begin{aligned}
& K_{11}(s, r)=K_{21}(s, r)=0, \\
& \left.K_{22}(s, r)=-\frac{A_{1}(r, s)}{\alpha_{1}(s)}, \quad \text { (throughout } J_{s r}\right) .
\end{aligned}
$$

Consequently $K(s, r)=0$ whenever the second argument $r$ is in the interval $J^{(1)}$. Let us write for short

$$
\boldsymbol{A}(s, r)=-\frac{A_{1}(r, s)}{\alpha_{1}(s)}, \quad \boldsymbol{B}(s, r)=-\frac{B_{1}(r, s)}{\beta_{1}(s)},
$$

and denote their resolvent functions, which by hypothesis exist, by $\mathbf{A}^{\prime}(s, r)$, $\mathbf{Z}^{\prime}(s, r)$. Then

$$
\begin{aligned}
\Delta & =\sum_{n=0}^{\infty} \frac{(-1)^{n}}{n !} \int_{J^{\prime}}^{(n)} \cdots \int_{J^{\prime}} K\left(\begin{array}{l}
s_{1} \cdots s_{n} \\
s_{1} \cdots s_{n}
\end{array}\right) d s_{1} \cdots d s_{n} \\
& =\sum_{n=0}^{\infty} \frac{(-1)^{n}}{n !} \int_{a}^{\beta} \cdots \int_{a}^{\beta} K_{22}\left(\begin{array}{l}
s_{1} \cdots s_{n} \\
s_{1} \cdots s_{n}
\end{array}\right) d s_{1} \cdots d s_{n} .
\end{aligned}
$$

This is different from zero, because it is precisely the Fredholm determinant of the kernel $\mathbf{A}(s, r)$. This completes the proof that $U_{2}$ is integro-linearly independent of $U_{1}$ by Theorem VI, $\S 4$.

From the resolvent relations (15), $\S 4$, we have also the following further facts:

$$
\begin{aligned}
& Q_{11}(s, r)=Q_{21}(s, r)=0, \\
& Q_{22}(s, r)=K_{22}(s, r)+\int_{\alpha}^{\beta} Q_{22}(s, t) K_{22}(t, r) d t, \\
& Q_{22}(s, r)=K_{22}(s, r)+\int_{a}^{\beta} K_{22}(s, t) Q_{22}(t, r) d t, \\
& Q_{12}(s, r)=K_{12}(s, r)+\int_{a}^{\beta} K_{12}(s, t) Q_{22}(t, r) d t,
\end{aligned}
$$

whence $Q_{22}(s, r)=\mathbf{A}^{\prime}(s, r)$. Furthermore,

$$
C_{1}(s, r)=-\frac{Q_{12}(s, r)}{\alpha_{1}(r)}, \quad D_{1}(s, r)=0 .
$$

If we let $\alpha_{1}(s)= \pm \beta_{1}(s)=1$, as we may do without loss of generality, we have

$$
\begin{aligned}
& \Delta(s)=1, \quad \mathbf{A}(s, r)=-A_{1}(r, s), \quad \mathbf{R}(s, r)=\mp B_{1}(r, s), \\
& K_{12}(s, r)= \pm \boldsymbol{Z}(s, r) \mp \mathbf{A}(s, r) \text {, } \\
& -C_{1}(s, r)=Q_{12}(s, r)=\mp \mathbf{A}^{\prime}(s, r) \pm \boldsymbol{B}(s, r) \pm \int_{a}^{\boldsymbol{\beta}} \boldsymbol{\beta}(s, t) \mathbf{A}^{\prime}(t, r) d t,
\end{aligned}
$$


and the equations $(3),\left(3^{\prime}\right)$ have the following form

Solving,

$$
\begin{aligned}
& N(s, r)=C_{1}(s, r) \pm \mathbf{A}(r, s)+\int_{a}^{\beta} \mathbf{A}(r, t) N(s, t) d t, \\
& N(s, r)= \pm(r, s)+\int_{a}^{\beta}(r, t) N(s, t) d t .
\end{aligned}
$$

$$
\begin{aligned}
N(s, r)= & {\left[C_{1}(s, r) \pm \mathbf{A}(r, s)\right]+\int_{a}^{\beta} \mathbf{A}^{\prime}(r, t)\left[C_{1}(s, t) \pm \mathbf{A}(t, s)\right] d t } \\
= & \pm\left[\mathbf{A}^{\prime}(s, r)+\mathbf{A}^{\prime}(r, s)+\int_{a}^{\beta} \mathbf{A}^{\prime}(s, t) \mathbf{A}^{\prime}(r, t) d t\right] \mp(s, r) \\
& \mp \int_{a}^{\beta}(s, \sigma)\left[\mathbf{A}^{\prime}(\sigma, r)+\mathbf{A}^{\prime}(r, \sigma)\right. \\
& \left.+\int_{a}^{\beta} \mathbf{A}^{\prime}(\sigma, t) \mathbf{A}^{\prime}(r, t) d t\right] d \sigma, \\
N(s, r)= & \pm(r, s) \pm \int_{a}^{\beta}(r, t) \pm(r, s) .
\end{aligned}
$$

Equating and transposing,

$$
\begin{aligned}
& {\left[\mathbf{A}^{\prime}(s, r)+\mathbf{A}^{\prime}(r, s)+\int_{a}^{\beta} \mathbf{A}^{\prime}(s, t) \mathbf{A}^{\prime}(r, t) d t\right]=\boldsymbol{z}(s, r)+\boldsymbol{z}^{\prime}(r, s)} \\
& +\int_{a}^{\beta}(s, \sigma)\left[\mathbf{A}^{\prime}(\sigma, r)+\mathbf{A}^{\prime}(r, \sigma)+\int_{a}^{\beta} \mathbf{A}^{\prime}(\sigma, t) \mathbf{A}^{\prime}(r, t) d t\right] d \sigma .
\end{aligned}
$$

Upon solving and simplifying, we obtain the last condition in the final form

$$
\begin{aligned}
\mathbf{A}^{\prime}(s, r)+\mathbf{A}^{\prime}(r, s) & +\int_{\alpha}^{\beta} \mathbf{A}^{\prime}(s, \sigma) \mathbf{A}^{\prime}(r, \sigma) d \sigma \\
= & \boldsymbol{B}^{\prime}(s, r)+\boldsymbol{B}^{\prime}(r, s)+\int_{\alpha}^{\beta} \boldsymbol{B}^{\prime}(s, \sigma) \mathbf{B}^{\prime}(r, \sigma) d \sigma .
\end{aligned}
$$

TheOREM I. Every self-adjoint integro-linear boundary condition may be reduced to the form

$$
\begin{array}{r}
U[u ; s] \equiv u(a, s) \pm u(b, s)-\int_{a}^{\beta}[u(a, r) \mathbf{A}(r, s) \\
\pm u(b, r) \boldsymbol{Z}(r, s)] d r=0,
\end{array}
$$

in which the Fredholm determinants of and are not zero and their resolvent functions $\mathbf{A}^{\prime}$ and satisfy the relation (8). Conversely, every condition of this form is self-adjoint, provided, of course, that the condition $(C)$

is fulfilled.

$$
1 \pm R\left(\begin{array}{l}
b s \\
a
\end{array}\right) \neq 0
$$


COROLLARY. When the integro-differential expression $L[u]$ is anti-self-adjoint and the boundary condition $U_{1}[u]=0$ is self-adjoint, the latter must have the form

$$
\begin{aligned}
U[u] \equiv u(a, s)+u(b, s)-\int_{a}^{\beta}[u(a, r) \mathbf{A}(r, s) & \\
& +u(b, r) \mathbf{z}(r, s)] d r=0 .
\end{aligned}
$$

For, when $L[u]$ is anti-self-adjoint, $R\left(\begin{array}{c}x^{8} \\ y^{8}\end{array}\right) \equiv 1$; so the condition $(C)$ will not be satisfied when $\alpha(s)=-\beta(s)=1$.

\section{The Green's Functions}

In the theory of linear differential equations the conception of the Green's functions enables us to write down in an explicit form the solution of a semihomogeneous boundary problem consisting of a single linear differential equation of the $n$th order, or a system of $n$ linear differential equations of the first order, and of a system of $n$ homogeneous linear boundary equations, whenever the reduced system is incompatible.*

Following out this analogy, we are led to try to find a solution of a system

$$
L[u]=\lambda(x, s), \quad U[u]=0
$$

in the form

(1) $u(x, s)=\int_{a}^{a b} H\left(\begin{array}{l}x s \\ y\end{array}\right) \lambda(y, s) d y+\int_{a}^{b} \int_{a}^{\beta} G\left(\begin{array}{l}x s \\ y t\end{array}\right) \lambda(y, t) d t d y$,

where $H$ and $G$ are independent of $\lambda$. These two functions we shall call the system of Green's functions for $\left(A, B_{0}\right)$. We may arrive at such functions by imposing certain conditions of discontinuity suggested by the discontinuities of Green's functions for differential equations.

Let $G\left(\begin{array}{ll}x & s \\ y & i\end{array}\right)$ be continuous, together with its first partial derivative with respect to $x$, throughout the region $I_{x y} J_{s t}$; let $H\left(\begin{array}{l}x s \\ y\end{array}\right)$ be continuous, together with its first partial derivative with respect to $x$, throughout each of the following regions:

$$
T_{1}: \quad\left\{a \leqq y \leqq x \leqq b, J_{s}\right\}, \quad T_{2}: \quad\left\{a \leqq x \leqq y \leqq b, J_{s}\right\} ;
$$

finally let $H$ possess a discontinuity when $x=y$ of the type

$$
H\left(\begin{array}{l}
y+, s \\
y
\end{array}\right)-H^{I}\left(\begin{array}{l}
y-, s \\
y
\end{array}\right)=1 .
$$

* Birkhoff, these Trans a c t i ons, vol. 9 (1908), p. 377 ; Bounitzky, L i o u ville's Journal, ser. 6, vol. 5 (1909), p. 65 ; Bôcher, Annals of Mathematics, ser. 2, vol. 13 (1911-12), p. 71. 
We use the notation $H\left(\begin{array}{l}y \pm, s \\ y\end{array}\right)$ to mean $\lim _{\epsilon \doteq 0} H,\left(\begin{array}{l}y \pm \epsilon, s \\ y\end{array}\right) \epsilon>0$; also

$$
H\left(\begin{array}{l}
y \\
y \pm
\end{array}\right)=\lim _{\epsilon=0} H\left(\begin{array}{l}
x \\
x \pm \epsilon
\end{array}\right)
$$

Because of the continuity throughout $T_{1}$ and $T_{2}$, it is clear that

$$
H\left(\begin{array}{l}
y \pm, s \\
y
\end{array}\right)=H\left(\begin{array}{lr}
y & s \\
y
\end{array}\right) .
$$

Replacing $u$ by its value from (1), we find

$$
\begin{aligned}
& L[u]=\left[H\left(\begin{array}{l}
x \\
x
\end{array}\right)-H\left(\begin{array}{l}
x \\
x+
\end{array}\right)\right] \lambda(x, s) \\
&+\int_{a}^{b}\left[\frac{\partial H\left(\begin{array}{l}
x s \\
y
\end{array}\right)}{\partial x}+\phi(x, s) H\left(\begin{array}{l}
x s \\
y
\end{array}\right)\right] \lambda(y, s) d y \\
&+\int_{a}^{b} \int_{a}^{\beta}\left[L\left[G\left(\begin{array}{l}
x s \\
y t
\end{array}\right)\right]+\psi\left(\begin{array}{c}
s \\
x t
\end{array}\right) H\left(\begin{array}{l}
x t \\
y
\end{array}\right)\right] \lambda(y, t) d t d y .
\end{aligned}
$$

Hence, on account of (2), $u$ as given by (1) satisfies ( $A$ ) for every continuous function $\lambda(x, s)$ if and only if $H\left(\begin{array}{c}x s \\ y\end{array}\right)$ and $G\left(\begin{array}{l}x \\ y\end{array}\right)$ respectively satisfy the equations

$$
\begin{gathered}
\frac{\partial H\left(\begin{array}{l}
x s \\
y
\end{array}\right)}{\partial x}+\phi(x, s) H\left(\begin{array}{l}
x s \\
y
\end{array}\right)=0 \\
L\left[G\left(\begin{array}{l}
x s \\
y
\end{array}\right)\right]=-\psi\left(\begin{array}{l}
s \\
x
\end{array}\right) H\left(\begin{array}{l}
x t \\
y
\end{array}\right) .
\end{gathered}
$$

Both of these equations have to be considered separately in the regions $T_{1}$ and $T_{2}$, although the formal work is the same.

We may now regard the functions

$$
H\left(\begin{array}{l}
y+, s \\
y
\end{array}\right), H\left(\begin{array}{l}
y-, s \\
y
\end{array}\right), G\left(\begin{array}{l}
y s \\
y t
\end{array}\right)
$$

as the initial functions given at a fixed point $y$ in the interval $I_{x}$. These functions will at present be assumed to be continuous in their respective variables, and to satisfy condition (2); otherwise they are arbitrary, pending further determination.

By (2), $\S 2$, the solution of (3) is

$$
H\left(\begin{array}{l}
x s \\
y
\end{array}\right)=R\left(\begin{array}{l}
x s \\
y
\end{array}\right) H\left(\begin{array}{l}
y \pm, s \\
y
\end{array}\right),
$$

in which the \pm signs correspond respectively to the regions $T_{1}, T_{2}$. 
The equation (4) may be solved by the result of $\S 2$ in the form

$$
\begin{aligned}
G\left(\begin{array}{l}
x s \\
y
\end{array}\right) & =R\left(\begin{array}{l}
x s \\
y
\end{array}\right) G\left(\begin{array}{l}
y s \\
y
\end{array}\right)+\int_{a}^{\beta} S\left(\begin{array}{l}
x s \\
y \sigma
\end{array}\right) G\left(\begin{array}{l}
y \sigma \\
y t
\end{array}\right) d \sigma \\
& -\int_{y}^{x}\left[R\left(\begin{array}{l}
x s \\
\xi
\end{array}\right) \psi\left(\begin{array}{c}
s \\
\xi t
\end{array}\right) H\left(\begin{array}{l}
\xi t \\
y
\end{array}\right)+\int_{a}^{\beta} S\left(\begin{array}{l}
x s \\
\xi \sigma
\end{array}\right) \psi\left(\begin{array}{c}
\sigma \\
\xi t
\end{array}\right) H\left(\begin{array}{l}
\xi t \\
y
\end{array}\right) d \sigma\right] d \xi .
\end{aligned}
$$

By Corollary III, Theorem I, $\S 2$, this simplifies into

$$
\begin{aligned}
& G\left(\begin{array}{l}
x s \\
y t
\end{array}\right)=R\left(\begin{array}{l}
x s \\
y
\end{array}\right) G\left(\begin{array}{l}
y s \\
y t
\end{array}\right)+\int_{a}^{\beta} S\left(\begin{array}{l}
x s \\
y \sigma
\end{array}\right) G\left(\begin{array}{l}
y \sigma \\
y t
\end{array}\right) d \sigma \\
& +\int_{y}^{x} \theta\left(\begin{array}{l}
x s \\
\xi t
\end{array}\right) H\left(\begin{array}{l}
\xi t \\
y
\end{array}\right) d \xi .
\end{aligned}
$$

Replacing $H\left(\begin{array}{c}\xi \\ y\end{array}\right)$ by its values from (5) and making use of the definition (10), $\S 2$, this further simplifies into

$$
\begin{aligned}
& G\left(\begin{array}{ll}
x & s \\
y & t
\end{array}\right)=R\left(\begin{array}{l}
x s \\
y
\end{array}\right) G\left(\begin{array}{l}
y s \\
y t
\end{array}\right) \\
& +\int_{a}^{\beta} S\left(\begin{array}{l}
x s \\
y \sigma
\end{array}\right) G\left(\begin{array}{l}
y \sigma \\
y t
\end{array}\right) d \sigma+H\left(\begin{array}{l}
y \pm, t \\
y
\end{array}\right) S\left(\begin{array}{l}
x s \\
y t
\end{array}\right),
\end{aligned}
$$

where the \pm signs again correspond to the regions $T_{1}$ and $T_{2}$. It is important to observe that the function $G\left(\begin{array}{ll}x & 8 \\ y & t\end{array}\right)$ thus determined is continuous throughout $I_{x y} J_{s t}$, because the only possible place of discontinuity is when $x=y$, but then $S\left(\begin{array}{ll}x & 8 \\ y & t\end{array}\right)=0$ by virtue of its definition.

We are now to determine $H\left(\begin{array}{c}y \pm, 8 \\ y\end{array}\right)$ and $G\left(\begin{array}{l}y \\ y \\ y\end{array}\right)$ so that the expression (1) also satisfies the boundary equation $\left(B_{0}\right)$ for all $\lambda(x, s)$. Upon substitution of (1) in $U[u]$ we have

$$
\begin{aligned}
U[u]=\int_{a}^{o b}\left[\alpha(s) H\left(\begin{array}{l}
a s \\
y
\end{array}\right)+\right. & \left.\beta(s) H\left(\begin{array}{l}
b s \\
y
\end{array}\right)\right] \lambda(y, s) d y \\
& +\int_{a}^{b} \int_{a}^{\beta}\left[A(s t) H\left(\begin{array}{l}
a t \\
y
\end{array}\right)+B(s t) H\left(\begin{array}{l}
b t \\
y
\end{array}\right)\right. \\
& \left.+U\left[G\left(\begin{array}{l}
x s \\
y
\end{array}\right)\right]\right] \lambda(y, t) d t d y .
\end{aligned}
$$

We shall have $U[u]=0$ for all $\lambda(x, s)$ if and only if the equations

$$
\begin{gathered}
\alpha(s) H\left(\begin{array}{l}
a s \\
y
\end{array}\right)+\beta(s) H\left(\begin{array}{l}
b s \\
y
\end{array}\right)=0 \\
U\left[G\left(\begin{array}{l}
x s \\
y t
\end{array}\right)\right]+A(s t) H\left(\begin{array}{l}
a t \\
y
\end{array}\right)+B(s t) H\left(\begin{array}{l}
b t \\
y
\end{array}\right)=0
\end{gathered}
$$

are satisfied. 
On substituting in (7) for $H\left(\begin{array}{c}a \\ y\end{array}\right)$ and $H\left(\begin{array}{c}b \\ y^{\circ}\end{array}\right)$ their values from (5), wc obtain an equation in $H\left(y_{y}^{y+,}\right)$ and $H\left(y_{y}^{y-, 8}\right)$, which together with (2) enables us to find for these functions the values

(9) $H\left(\begin{array}{l}y+, s \\ y\end{array}\right)=\frac{\alpha(s) R\left(\begin{array}{l}a s \\ y\end{array}\right)}{g(y, s)}, \quad H\left(\begin{array}{l}y-, s \\ y\end{array}\right)=\frac{-\beta(s) R\left(\begin{array}{l}b s \\ y\end{array}\right)}{g(y, s)}$,

since we confine ourselves to the case $g(y, s) \neq 0(\S 4)$. It is convenient at this stage to introduce the following abbreviations which will be useful later.

$$
g_{1}(y, s)=\alpha(s) R\left(\begin{array}{l}
a s \\
y
\end{array}\right), \quad g_{2}(y, s)=\beta(s) R\left(\begin{array}{l}
b s \\
y
\end{array}\right),
$$

$$
\begin{aligned}
& G_{1}\left(\begin{array}{c}
s \\
y t
\end{array}\right)=A(s, t) R\left(\begin{array}{l}
a t \\
y
\end{array}\right)+\alpha(s) S\left(\begin{array}{l}
a s \\
y t
\end{array}\right)+\int_{a}^{\beta} A(s, \sigma) S\left(\begin{array}{l}
a \sigma \\
y t
\end{array}\right) d \sigma \\
& G_{2}\left(\begin{array}{c}
s \\
y t
\end{array}\right)=B(s, t) R\left(\begin{array}{l}
b t \\
y
\end{array}\right)+\beta(s) S\left(\begin{array}{l}
b s \\
y
\end{array}\right)+\int_{\alpha}^{\beta} B(s, \sigma) S\left(\begin{array}{l}
b \sigma \\
y t
\end{array}\right) d \sigma
\end{aligned}
$$

Thus according to the notations $(6), \S 3$, we have*

$$
g(y, s)=g_{1}(y, s)+g_{2}(y, s), \quad G\left(\begin{array}{c}
s \\
y t
\end{array}\right)=G_{1}\left(\begin{array}{r}
s \\
y t
\end{array}\right)+G_{2}\left(\begin{array}{c}
s \\
y t
\end{array}\right),
$$

and the equations (9) may be written

$$
H\left(\begin{array}{l}
y+, s \\
y
\end{array}\right)=\frac{g_{1}(y, s)}{g(y, s)}, \quad H\left(\begin{array}{l}
y-, s \\
y
\end{array}\right)=-\frac{g_{2}(y, s)}{g(y, s)} .
$$

Now from the equation (6) we have

$$
\begin{aligned}
& G\left(\begin{array}{l}
a s \\
y t
\end{array}\right)=R\left(\begin{array}{l}
a s \\
y
\end{array}\right) G\left(\begin{array}{l}
y s \\
y t
\end{array}\right) \\
& +\int_{a}^{\beta} S\left(\begin{array}{l}
a s \\
y \sigma
\end{array}\right) G\left(\begin{array}{l}
y \sigma \\
y t
\end{array}\right) d \sigma+H\left(\begin{array}{l}
y-, t \\
y
\end{array}\right) S\left(\begin{array}{l}
a s \\
y t
\end{array}\right), \\
& G\left(\begin{array}{l}
b s \\
y t
\end{array}\right)=R\left(\begin{array}{l}
b s \\
y
\end{array}\right) G\left(\begin{array}{l}
y s \\
y t
\end{array}\right) \\
& +\int_{a}^{\beta} S\left(\begin{array}{l}
b s \\
y \sigma
\end{array}\right) G\left(\begin{array}{l}
y \sigma \\
y t
\end{array}\right) d \sigma+H\left(\begin{array}{l}
y+, t \\
y
\end{array}\right) S\left(\begin{array}{l}
b s \\
y t
\end{array}\right) ;
\end{aligned}
$$

* Note that $G\left(\begin{array}{c}s \\ y t\end{array}\right)$ and $G\left(\begin{array}{c}x s \\ y t\end{array}\right)$ are two entirely different functions. 
whence

$$
\begin{aligned}
U\left[G\left(\begin{array}{l}
x s \\
y t
\end{array}\right)\right]= & g(y, s) G\left(\begin{array}{l}
y s \\
y
\end{array}\right)+\int_{\alpha}^{\beta} G\left(\begin{array}{l}
s \\
y r
\end{array}\right) G\left(\begin{array}{l}
y r \\
y
\end{array}\right) d r \\
& +H\left(\begin{array}{l}
y-, t \\
y
\end{array}\right)\left[\alpha(s) S\left(\begin{array}{l}
a s \\
y
\end{array}\right)+\int_{\alpha}^{\beta} A(s, \sigma) S\left(\begin{array}{l}
a \sigma \\
y t
\end{array}\right) d \sigma\right] \\
& +H\left(\begin{array}{l}
y+, t \\
y
\end{array}\right)\left[\beta(s) S\left(\begin{array}{l}
b s \\
y
\end{array}\right)+\int_{\alpha}^{\beta} B(s, \sigma) S\left(\begin{array}{l}
b \sigma \\
y t
\end{array}\right) d \sigma\right] .
\end{aligned}
$$

We shall now substitute this value in (8) and also replace $H\left(\begin{array}{l}a t \\ y\end{array}\right), H\left(\begin{array}{l}b \\ y\end{array}\right)$ by their values from (5). If in the resulting equation we replace $H\left(y_{y}^{y+8}\right)$, $H\left(y_{y}^{y-, 8}\right)$ by their values from $\left(9^{\prime}\right)$, we find that (8) reduces to

$$
\begin{aligned}
g(y, s) G\left(\begin{array}{l}
y s \\
y t
\end{array}\right)+\int_{\alpha}^{\beta} G\left(\begin{array}{r}
s \\
y r
\end{array}\right) G\left(\begin{array}{l}
y r \\
y t
\end{array}\right) d r \\
=\frac{1}{g(y, t)}\left[g_{2}(y, t) G_{1}\left(\begin{array}{r}
s \\
y t
\end{array}\right)-g_{1}(y, t) G_{2}\left(\begin{array}{r}
s \\
y t
\end{array}\right)\right],
\end{aligned}
$$

or

$$
G\left(\begin{array}{l}
y s \\
y t
\end{array}\right)=F\left(\begin{array}{r}
s \\
y t
\end{array}\right)+\int_{a}^{\beta} K\left(\begin{array}{r}
s \\
y r
\end{array}\right) G\left(\begin{array}{l}
y r \\
y t
\end{array}\right) d r,
$$

if we write for short

$$
F\left(\begin{array}{r}
s \\
y t
\end{array}\right)=\frac{-1}{g(y, s) g(y, t)}\left|\begin{array}{lr}
g_{1}(y, t) & G_{1}\left(\begin{array}{r}
s \\
y t
\end{array}\right) \\
g_{2}(y, t) & G_{2}\left(\begin{array}{r}
s \\
y t
\end{array}\right)
\end{array}\right| \text {. }
$$

The kernel $\left.K{ }_{y \cdot \dot{r}}{ }^{*}\right)$ is the same as that in the equation $\left(7^{\prime}\right), \S 3$.

Now if the homogeneous system $\left(A_{0}, B_{0}\right)$ is incompatible, then the kernel

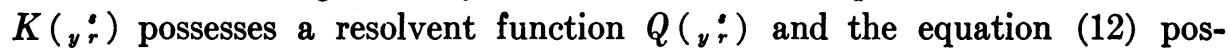
sesses a unique solution given by

$$
G\left(\begin{array}{l}
y s \\
y t
\end{array}\right)=F\left(\begin{array}{r}
s \\
y t
\end{array}\right)+\int_{a}^{\beta} Q\left(\begin{array}{r}
s \\
y r
\end{array}\right) F\left(\begin{array}{r}
r \\
y t
\end{array}\right) d r .
$$

Definition. The functions $H\left(\begin{array}{c}x s \\ y\end{array}\right), G\left(\begin{array}{cc}x & 8 \\ y & t\end{array}\right)$ are said to form a system of Green's functions of the integro-differential boundary problem $\left(A_{0}, B_{0}\right)$ : $L[u]=0, U[u]=0$, where $U[u]$ is assumed to be integro-linearly selfindependent and subject to the condition $(C)$, if they are defined respectively in the regions $I_{x y} J_{s}$ and $I_{x y} J_{s t}$ and possess the following properties:

1. $H\left(\begin{array}{l}x x^{\circ} \\ y^{\prime}\end{array}\right)$ is continuous together with the first partial derivative with respect to $x$ in the regions $T_{1}$ and $T_{2}$, and

$$
H\left(\begin{array}{l}
y+, s \\
y
\end{array}\right)-H\left(\begin{array}{l}
y-, s \\
y
\end{array}\right)=1 .
$$


2. $G\left(\begin{array}{ll}x & s \\ y & t\end{array}\right)$ is continuous together with the first partial derivative with respect to $x$ throughout the region $I_{x y} J_{s t}$.

3. Throughout $T_{1}$ and $T_{2}$ the functions $H\left(\begin{array}{c}x^{\prime} \\ y\end{array}\right)$ satisfies the equations (3) and (7).

4. The function $G\left(\begin{array}{cc}x & 8 \\ y & t\end{array}\right)$ satisfies the equations (4) and (8).

Theorem I. When Green's functions exist, the semi-homogeneous system $\left(A, B_{0}\right)$ possesses a solution given by the formula (1).

We have seen in the above deduction that Green's functions exist if the system $\left(A_{0}, B_{0}\right)$ is incompatible. Because of the fact (Theorem III, $\S 4$ ) that when $\left(A_{0}, B_{0}\right)$ is compatible not every semi-homogeneous system ( $A$, $B_{0}$ ) can have a solution, it follows that Green's functions do not exist for this case. Hence

'THEOREM II. A necessary and sufficient condition that a system of Green's functions exist for a system $\left(A_{0}, B_{0}\right)$, in which $U$ is self-independent and $(C)$ is fulfilled, is that the system $\left(A_{0}, B_{0}\right)$ be incompatible. When this condition is satisfied, the solution given by (1) is the unique solution.

The last fact follows from the Corollary to Theorem III, §3. From the theorem just stated, it follows that the equation (12) cannot possess a solution whenever the system $\left(A_{0}, B_{0}\right)$ is compatible. Thus we have the

Corollary. When a system $\left(A_{0}, B_{0}\right)$, in which $U$ is self-independent and fulfills $(C)$, is compatible, the function (13) cannot vanish identically; and

$$
\int_{a}^{\beta} \phi_{i}(y, s) F\left(\begin{array}{r}
s \\
y
\end{array}\right) d s
$$

does not vanish identically for every $\phi_{i}(y, s)$ which satisfies

$$
\phi(y, s)=\int_{a}^{\beta} \phi(y, r) K\left(\begin{array}{r}
r \\
y s
\end{array}\right) d r .
$$

Theorem III. For a system $\left(A, B_{0}\right)$ there cannot exist more than one set of functions $H\left(\begin{array}{ll}x & s \\ y\end{array}\right), G\left(\begin{array}{ll}x & s \\ y & t\end{array}\right)$ such that (1) is a solution of the system for every $\lambda(x, s)$; and if such a set exists, it consists of the Green's functions for the system.

When the reduced system $\left(A_{0}, B_{0}\right)$ is compatible, no such functions $H$ and $G$ can exist, because in that case not every semi-homogeneous system $\left(A, B_{0}\right)$ can have a solution (Theorem III, $\left.\S 4\right)$. When $\left(A_{0}, B_{0}\right)$ is incompatible, Green's functions exist and (1) is the unique solution of $\left(A, B_{0}\right)$. Hence if there exists another set of functions, $H^{\prime}$ and $G^{\prime}$, such that

$$
u(x, s)=\int_{a}^{b} H^{\prime}\left(\begin{array}{l}
x s \\
y
\end{array}\right) \lambda(y, s) d y+\int_{a}^{b} \int_{\alpha}^{\beta} G^{\prime}\left(\begin{array}{l}
x s \\
y
\end{array}\right) \lambda(y, t) d t d y
$$

is also a solution of $\left(A, B_{0}\right)$, this solution must be identical with (1) and 
therefore the difference of this and (1) is identically zero. Since $\lambda(x, s)$ is arbitrary, we find, by using the lemma in $\S 5, H^{\prime} \equiv H, G^{\prime} \equiv G$.

. If in the system $\left(A, B_{0}\right)$ we replace the boundary condition $U=0$ by another boundary condition $U^{\prime}=0$, where $U^{\prime}$ is an integro-linear function of $U$, then (1) will be obviously also a solution of the resulting system; hence

CoRollary. The Green's functions of a system are invariant of the choice of boundary conditions, provided the different choices of boundary expressions are integro-linearly connected.

Another important property is that there exists a symmetrical relation between the Green's functions of the given system and the adjoint system. From Corollary I, Theorem I, $\$ 6$, it follows that the adjoint boundary condition $V=0$ is self-independent. By reference to Theorems II, III, $\S 7$, we infer from Theorem II:

Theorem IV. If the system $\left(A_{0}, B_{0}\right)$ possesses Green's functions, $H, G$, the adjoint system $\left(\bar{A}_{0}, \bar{B}_{0}\right)$ possesses Green's functions, $\bar{H}, \bar{G}$.

The solution of the adjoint semi-homogeneous system

$$
\left(\bar{A}, \overline{B_{0}}\right) \quad-M[v]=\mu(x, s), \quad V[v]=0
$$

is given by

(15) $v(x, s)=\int_{a}^{b} \bar{H}\left(\begin{array}{l}x s \\ y\end{array}\right) \mu(y, s) d y+\int_{a}^{b} \int_{a}^{\beta} \bar{G}\left(\begin{array}{l}x s \\ y t\end{array}\right) \mu(y, t) d t d y$.

Let $u(x, s)$ be the solution of the system $\left(A, B_{0}\right)$ given by (1). Then, by Green's theorem,

$$
\int_{a}^{b} \int_{a}^{\beta}[v(x, s) \lambda(x, s)+u(x, s) \mu(x, s)] d s d x=0 .
$$

On the substitution of the values of $u$ and $v$ from (1) and (15), we have

$$
\begin{aligned}
& \int_{a}^{b} \int_{a}^{b} \int_{a}^{\beta}\left[\bar{H}\left(\begin{array}{l}
x s \\
y
\end{array}\right)+H\left(\begin{array}{l}
y s \\
x
\end{array}\right)\right] \lambda(x, s) \mu(y, s) d s d y d x
\end{aligned}
$$

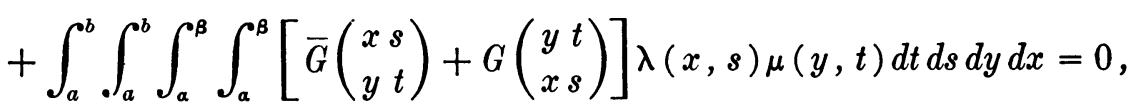

which holds for every $\lambda$ and $\mu$. Hence, by the lemma in $\S 5$,

$$
\bar{H}\left(\begin{array}{l}
x s \\
y
\end{array}\right)=-H\left(\begin{array}{l}
y s \\
x
\end{array}\right), \quad \bar{G}\left(\begin{array}{l}
x s \\
y t
\end{array}\right)=-G\left(\begin{array}{l}
y t \\
x s
\end{array}\right) .
$$

Theorem V. The Green's functions of adjoint systems satisfy (16).

Theorem VI. If two systems

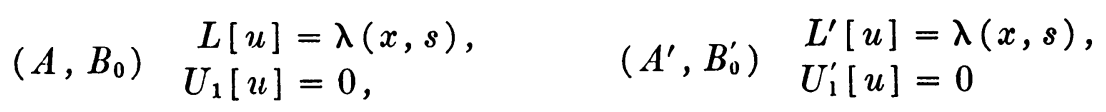


have the same Green's functions $H\left(\begin{array}{l}x \\ y\end{array}\right), G\left(\begin{array}{l}x \\ y \\ y\end{array}\right)$, and if the adjoint system $\left(\bar{A}_{0}, \bar{B}_{0}\right)$ exists, then the expressions $L$ and $L^{\prime}$ are identical and $U_{1}^{\prime}$ is an integrolinear function of $U_{1}$.

Since the Green's functions are the same for both systems, the function formed from them by the formula (1) satisfies both systems, hence it satisfies the homogeneous equation

that is,

$$
L[u]-L^{\prime}[u]=0,
$$

$$
\phi^{\prime \prime}(x, s) u(x, s)+\int_{\alpha}^{\beta} \psi^{\prime \prime}\left(\begin{array}{r}
x s \\
t
\end{array}\right) u(x, t) d t=0,
$$

if we let

$$
\begin{aligned}
\phi^{\prime \prime}(x, s) & =\phi(x, s)-\phi^{\prime}(x, s), \\
\psi^{\prime \prime}\left(\begin{array}{r}
s \\
x t
\end{array}\right) & =\psi\left(\begin{array}{r}
s \\
x t
\end{array}\right)-\psi^{\prime}\left(\begin{array}{r}
s \\
x t
\end{array}\right) .
\end{aligned}
$$

If we substitute (1) in this equation, we find

$$
\begin{aligned}
\int_{a}^{b} \phi^{\prime \prime}(x, s) H\left(\begin{array}{l}
x s \\
y
\end{array}\right) \lambda & (y, s) d y \\
& +\int_{a}^{b} \int_{a}^{\beta}\left[\phi^{\prime \prime}(x, s) G\left(\begin{array}{l}
x s \\
y t
\end{array}\right)+\psi^{\prime \prime}\left(\begin{array}{r}
s \\
x t
\end{array}\right) H\left(\begin{array}{l}
x t \\
y
\end{array}\right)\right. \\
& \left.+\int_{a}^{\beta} \psi^{\prime \prime}\left(\begin{array}{c}
s \\
x r
\end{array}\right) G\left(\begin{array}{l}
x r \\
y t
\end{array}\right) d r\right] \lambda(y, t) d t d y=0 .
\end{aligned}
$$

By the lemma of $\S 5$, we obtain

$$
\begin{gathered}
\phi^{\prime \prime}(x, s) H\left(\begin{array}{l}
x s \\
y
\end{array}\right) \equiv 0 \\
\phi^{\prime \prime}(x, s) G\left(\begin{array}{l}
x s \\
y t
\end{array}\right)+\psi^{\prime \prime}\left(\begin{array}{c}
s \\
x t
\end{array}\right) H\left(\begin{array}{l}
x t \\
y
\end{array}\right)+\int_{a}^{\beta} \psi^{\prime \prime}\left(\begin{array}{c}
s \\
x r
\end{array}\right) G\left(\begin{array}{l}
x r \\
y t
\end{array}\right) d r \equiv 0 .
\end{gathered}
$$

Let us take the limit of the first of these equations as $y$ approaches $x$ first from above and then from below. This gives

$$
\phi^{\prime \prime}(x, s) H\left(\begin{array}{l}
x \\
x \pm
\end{array}\right)=0,
$$

and by subtracting one of these equations from the other, we see from (2) that $\phi^{\prime \prime}=0$.

Substituting this value in the second equation (17), and replacing $H$ and $G$ by their values $-\bar{H},-\bar{G}$, we find

$$
\bar{H}\left(\begin{array}{l}
y t \\
x
\end{array}\right) \psi^{\prime \prime}\left(\begin{array}{c}
s \\
x t
\end{array}\right)+\int_{a}^{\beta} \bar{G}\left(\begin{array}{c}
y t \\
x r
\end{array}\right) \psi^{\prime \prime}\left(\begin{array}{c}
s \\
x r
\end{array}\right) d r \equiv 0 .
$$


Hence

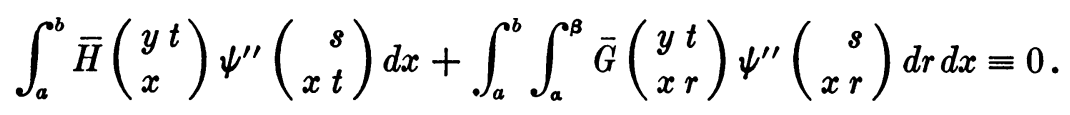

Now the first member of this equation is, by (15), the solution of the system

$$
-M[v]=\psi^{\prime \prime}\left(\begin{array}{r}
s \\
y t
\end{array}\right), \quad V_{1}[v]=0,
$$

regarded as equations in $y$ and $t$. Hence $\psi^{\prime \prime}=0$. This completes the proof that $L[u]$ and $L^{\prime}[u]$ are identical.

Our theorem will be proved if we can show that $U_{1}$ and $U_{1}^{\prime}$ are integrolinearly connected. For this purpose we substitute in $U_{1}^{\prime}$ for $u(a, s)$ and $u(b, s)$ their values from $(14), \S 6$. This gives

$$
\begin{aligned}
& U_{1}^{\prime}(s)=M_{1}(s) U_{1}(s)+\int_{a}^{\beta} N_{1}(s, t) U_{1}(t) d t \\
& \quad+M_{2}(s) U_{2}(s)+\int_{a}^{\beta} N_{2}(s, t) U_{2}(t) d t,
\end{aligned}
$$

in which

$$
\begin{aligned}
M_{2}(s)=-\alpha_{1}^{\prime}(s) \gamma_{1}(s)+ & \beta_{1}^{\prime}(s) \delta_{1}(s), \\
N_{2}(s, t)=-\alpha_{1}^{\prime}(s) C_{1}(t, s) & +\beta_{1}^{\prime}(s) D_{1}(t, s)-A_{1}^{\prime}(s, t) \gamma_{1}(t) \\
& +B_{1}^{\prime}(s, t) \delta_{1}(t)+\int_{a}^{\beta}\left[-A_{1}^{\prime}(s, r) C_{1}(t, r)\right. \\
& \left.+B_{1}^{\prime}(s, r) D_{1}(t, r)\right] d r .
\end{aligned}
$$

And if we can show that $M_{2}(s) \equiv 0, N_{2}(s, t) \equiv 0$, we shall have established an integro-linear relation between $U_{1}$ and $U_{1}^{\prime}$.

To prove $M_{2}(s) \equiv 0$, we make use of (7) and the corresponding formula for $\left(A^{\prime}, B_{0}^{\prime}\right)$. We have, since $\left(A, B_{0}\right)$ and $\left(A^{\prime}, B_{0}^{\prime}\right)$ have the same Green's functions,

$$
\begin{aligned}
& \alpha_{1}(s) H\left(\begin{array}{l}
a s \\
y
\end{array}\right)+\beta_{1}(s) H\left(\begin{array}{l}
b s \\
y
\end{array}\right)=0 \\
& \alpha_{1}^{\prime}(s) H\left(\begin{array}{l}
a s \\
y
\end{array}\right)+\beta_{1}^{\prime}(s) H\left(\begin{array}{l}
b s \\
y
\end{array}\right)=0 .
\end{aligned}
$$

Now for each constant value $s_{0}$ the functions $H\left(\begin{array}{l}a \\ y\end{array} 0^{0}\right)$ and $H\left(\begin{array}{ll}b & s_{0} \\ y\end{array}\right)$ cannot both vanish identically, because otherwise we would have from (5) both $H\left(\begin{array}{c}\left.y-, s_{0}\right) \\ y\end{array}\right)$ and $H\left(\begin{array}{c}y+, \\ y\end{array}, 0\right)$ identically zero, which is impossible owing to the discontinuity of $H$. Consequently

$$
-\alpha_{1}^{\prime}(s) \beta_{1}(s)+\beta_{1}^{\prime}(s) \alpha_{1}(s) \equiv 0 .
$$

Hence, from (8), $\S 6$, we have $M_{2} \equiv 0$. 
To show $N_{2}(s, t) \equiv 0$, we have from the formulæ corresponding to (8) in the case of the systems $\left(\bar{A}, \bar{B}_{0}\right)$ and $\left(A^{\prime}, B_{0}^{\prime}\right)$

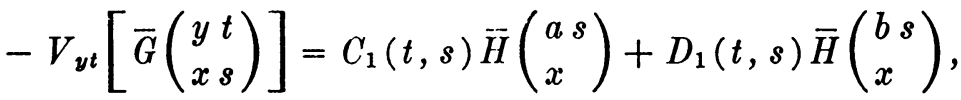

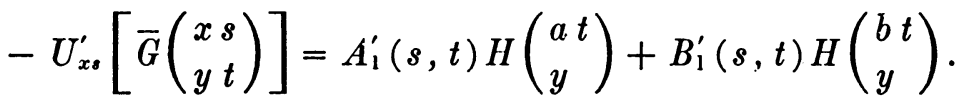

The subscript 1 has been dropped from $U_{1}$ and $V_{1}$ for convenience, and the variable subscripts are inserted to indicate the variables operated on. On account of the relation (16) we have the identity

$$
-U_{x s}^{\prime}\left[V_{y t}\left[\bar{G}\left(\begin{array}{l}
y t \\
x s
\end{array}\right)\right]\right] \equiv V_{y t}\left[U_{x s}^{\prime}\left[G\left(\begin{array}{l}
x s \\
y t
\end{array}\right)\right]\right],
$$

which may be written

$$
\begin{aligned}
U_{x \wedge}^{\prime}\left[C_{1}(t, s) \bar{H}\left(\begin{array}{l}
a s \\
x
\end{array}\right)\right. & \left.+D_{1}(t, s) \bar{H}\left(\begin{array}{l}
b s \\
x
\end{array}\right)\right] \\
& +V_{y t}\left[A_{1}^{\prime}(s, t) H\left(\begin{array}{l}
a t \\
y
\end{array}\right)+B_{1}^{\prime}(s, t) H\left(\begin{array}{l}
b t \\
y
\end{array}\right)\right] \equiv 0 .
\end{aligned}
$$

Expanding and collecting terms, we find

$$
\begin{aligned}
& {\left[\alpha_{1}^{\prime}(s) \ddot{H}\left(\begin{array}{l}
a s \\
a+
\end{array}\right)+\beta_{1}^{\prime}(s) \ddot{H}\left(\begin{array}{l}
a s \\
b
\end{array}\right)\right] C_{1}(t, s)+\left[\alpha_{1}^{\prime}(s) \ddot{H}\left(\begin{array}{l}
b s \\
a
\end{array}\right)\right.} \\
& \left.+\beta_{1}^{\prime}(s) \bar{H}\left(\begin{array}{ll}
b & s \\
b-
\end{array}\right)\right] D_{1}(t, s)+\left[\gamma_{1}(t) H\left(\begin{array}{l}
a \\
a+
\end{array}\right)\right. \\
& \left.+\delta_{1}(t) H\left(\begin{array}{l}
a t \\
b
\end{array}\right)\right] A_{1}^{\prime}(s, t)+\left[\gamma_{1}(t) H\left(\begin{array}{l}
b t \\
a
\end{array}\right)\right. \\
& \left.+\delta_{1}(t) H\left(\begin{array}{ll}
b & t \\
b & -
\end{array}\right)\right] B_{1}^{\prime}(s, t)+\int_{a}^{\beta}\left\{A _ { 1 } ^ { \prime } ( s , r ) C _ { 1 } ( t , r ) \left[\bar{H}\left(\begin{array}{lr}
a & r \\
a+
\end{array}\right)\right.\right. \\
& \left.\left.+H\left(\begin{array}{l}
a \\
a+
\end{array}\right)\right]+B_{1}^{\prime}(s, r) D_{1}(t, r)\left[\bar{H}\left(\begin{array}{lr}
b & r \\
b & -
\end{array}\right)+H\left(\begin{array}{ll}
b & r \\
b & -
\end{array}\right)\right]\right\} d r \\
& +\int_{a}^{\beta}\left\{A_{1}^{\prime}(s, r) D_{1}(t, r)\left[\bar{H}\left(\begin{array}{l}
b r \\
a
\end{array}\right)+H\left(\begin{array}{l}
a r \\
b
\end{array}\right)\right]\right. \\
& \left.+B_{1}^{\prime}(s, r) C_{1}(t, r)\left[\bar{H}\left(\begin{array}{l}
a r \\
b
\end{array}\right)+H\left(\begin{array}{l}
b r \\
a
\end{array}\right)\right]\right\} d r \equiv 0
\end{aligned}
$$

By means of the relations

$$
H\left(\begin{array}{l}
y \pm, s \\
y
\end{array}\right)=H\left(\begin{array}{ll}
y & s \\
y \mp
\end{array}\right)
$$


and (7), (16), (4), the first member of this equation reduces precisely to the expression $N_{2}(s, t)$. Thus our proof is completed.

CoROLlary. A necessary and sufficient condition that the Green's functions of a system be skew-symmetric, i. e.,

$$
H\left(\begin{array}{l}
x s \\
y
\end{array}\right)=-H\left(\begin{array}{l}
y s \\
x
\end{array}\right), \quad G\left(\begin{array}{l}
x s \\
y t
\end{array}\right)=-G\left(\begin{array}{l}
y t \\
x s
\end{array}\right),
$$

is that the integro-differential expression $L[u]$ be anti-self-adjoint and the boundary condition $U[u]=0$ be self-adjoint.

The sufficiency of this theorem follows from Theorem IV, and the necessity from the theorem just proved.

HARVARD UNIVERSITY May, 1917 